\title{
Enhanced Interactions of Amino Acids and Nucleic Acid Bases with Bare Black Phosphorene Monolayer Mediated by Coadsorbed Species
}

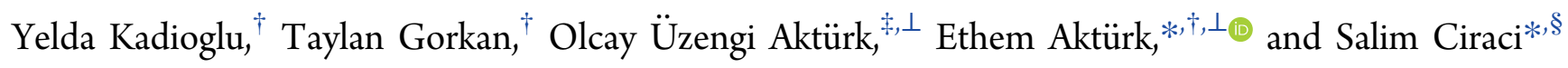

${ }^{\dagger}$ Department of Physics, Adnan Menderes University, Aydın 09100, Turkey

${ }^{\ddagger}$ Department of Electrical and Electronic Engineering, Adnan Menderes University, 09100 Aydın, Turkey

${ }^{\perp}$ Nanotechnology Application and Research Center, Adnan Menderes University, Aydın 09100, Turkey

${ }^{\S}$ Department of Physics, Bilkent University, Ankara 06800, Turkey

\section{Supporting Information}

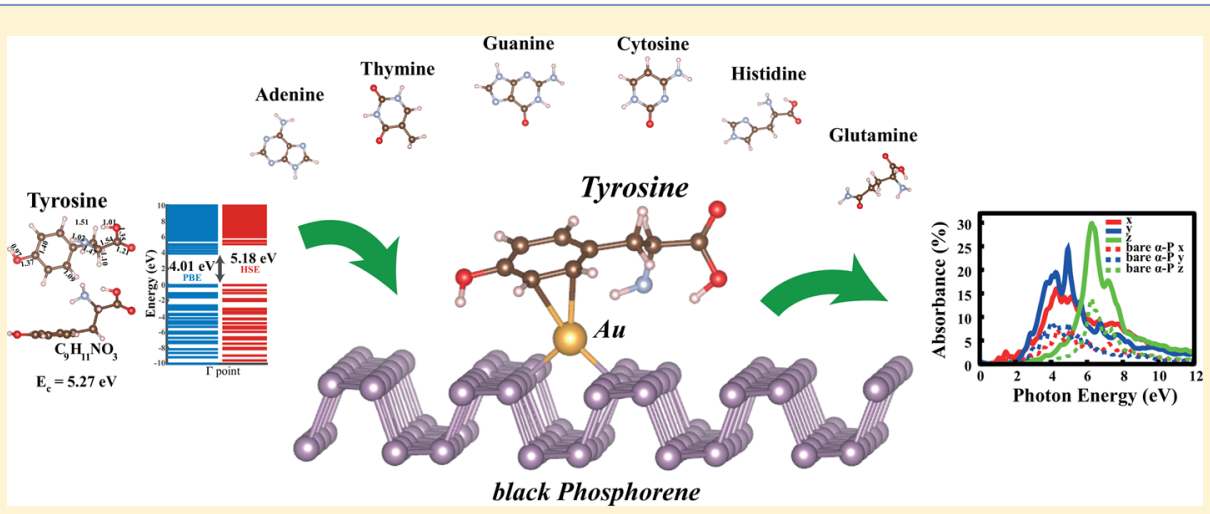

ABSTRACT: In this paper, we characterize amino acids and nucleic acid bases (nucleobases), such as glutamine, histidine, tyrosine, adenine, guanine, cytosine, and thymine, and examine their interaction with bare, as well as with gold cluster and $\mathrm{Ti}$ adatom covered, black phosphorene $(\alpha-\mathrm{P})$ monolayers using density functional theory. The binding of these amino acids and nucleobases to the bare $\alpha$-P monolayer is realized generally through weak van der Waals interaction and comprises only a small amount of charge exchange. Accordingly, the electronic energy structures of adsorbates and underlying substrate are not affected significantly. However, the electronic structure of bare $\alpha$-P is significantly affected upon adsorption of a gold cluster and a single $\mathrm{Ti}$ adatom; depending on the size of the adsorbate and the symmetry of their coverage, the energy band gap can be tuned and permanent magnetic moments can be attained. Additionally, the adsorption of amino acids or nucleobases to these adsorbates on an $\alpha$-P monolayer results in enhanced binding and hence makes their sustainable fixation on $\alpha$-P monolayer possible. In particular, a semiconducting Au decorated $\alpha$-P monolayer undergoes a metal-insulator transition upon the adsorption of tyrosine. This and similar effects favor the $\alpha$-P monolayer in biosensor applications. In contrast to the situation with adsorbates, the binding of amino acid is not enhanced when it adsorb to patterned vacancy or divacancy sites of the $\alpha$-P monolayer. Our study shows that the absorbance of the bare $\alpha$-P monolayer can be enhanced by coating with amino acid and nucleobases. The absorbance spectrum can be further modified by the adsorption of these molecules to gold atoms on the $\alpha$-P monolayer.

\section{INTRODUCTION}

Interfaces between biological molecules and two-dimensional (2D) inorganic nanostructures have been a subject of active research. $^{1-5}$ Through these interfaces, $2 \mathrm{D}$ materials can be functionalized for biointegrated electronics and biomedical applications like biosensors and smart drugs. ${ }^{6-9}$ Organic molecules that cover the surfaces of monolayers or multilayers of $2 \mathrm{D}$ materials can modify the electronic and optical properties of surfaces. ${ }^{10,11}$ Atomically thin $2 \mathrm{D}$ nanostructures like graphene can be utilized also as bare and flat templates for organic molecules to synthesize larger molecules. ${ }^{12,13}$ On the inorganic side, the interfaces with carbon nanotubes and graphene have been investigated actively owing to the chemical inertness, mechanical stability, and high surface-volume ratio. For cases requiring the fixation of organic molecules on the surface, patterned defects and adspecies can be created to ensure active sites to bind organic molecules to the surface. ${ }^{14-16}$ On the biological side, in particular, amino acids, which are basic elements of peptides, and deoxyribonu-

Received: May 18, 2019

Revised: July 31, 2019

Published: August 29, 2019 
cleic or ribonucleic acid (DNA/RNA) nucleobases are attracting growing interest.

DNA and polynucleotides are of vital importance due to genomics and diagnosis and treatment of diseases, particularly diverse cancers. DNA and organic molecule biosensors, which can be a biological recognition mechanism, play critical roles in detection of microorganisms or in diagnostics. ${ }^{17,18}$ In recent years, $2 \mathrm{D}$ materials have become crucial for biosensing devices. For example, graphene, silicene, monolayer $\mathrm{MoS}_{2}, \mathrm{WS}_{2}, \mathrm{~h}-\mathrm{BN}$, graphene nanoribbons, and silicon nanowires ${ }^{19-24}$ are found to be used for DNA base and larger amino acid detection. Therefore, studies on 2D materials for development of biosensing applications and DNA sequencing technology have become more popular in the last decades.

Amino acids have been analyzed by various experimental and theoretical techniques. ${ }^{25-28}$ Glycine has been investigated actively because it acts as an inhibitory neurotransmitter in the central nervous system in the brain. ${ }^{29}$ More recently, metalinsulator transition and heterostructure formation by glycine self-assembled on defect patterned graphene have been demonstrated. ${ }^{30}$ Beyond biological applications, it has been also predicted that the coverage of graphene by self-assembled glycine enhances solar spectrum absorbance significantly for photovoltaic applications. ${ }^{31}$ The interaction of some amino acids with a black phosphorene monolayer (i.e., a suspended monolayer of phosphorus in symmetric washboard structure, which is specified as $\alpha$-P) surface have been investigated ${ }^{3,4}$ and compared with that with the surface of graphene in honeycomb structure. It has been demonstrated that studies based on $a b$ initio density functional theory (DFT) are powerful and, at the same time, accurate to unveil the details of interaction between an organic molecule and a 2D flat surface of inorganic materials, which can affect electronic and optical properties.

In this work, we analyzed the interface of different types of amino acids and nucleic acid bases (nucleobases), glutamine, histidine, tyrosine, adenine, guanine, cytosine, and thymine, with the $2 \mathrm{D}$ bare black phosphorene monolayer, which will be named as $\alpha$-P in the rest of text. Each of these amino acids and nucleobases treated here gained importance and attracted considerable research interest as outlined in the forthcoming sections. Tyrosine, in particular, became well-known due to signal transduction mechanisms in the cell within phosphorylation by tyrosine kinase. ${ }^{32-34}$ This process affects immune, neuronal, cardiovascular, and metabolic systems of the body. Tyrosine is also an essential amino acid for production of basic neurotransmitters like dopamine, epinephrine, and norepinephrine. ${ }^{35,36}$ Therefore, we placed much emphasis on tyrosine adsorption on $\alpha$-P.

As for black phosphorene, it is used as a template for the self-assembly of DNA/RNA constituents. As compared to graphene, organic molecules on $\alpha$-P undergo relatively less deterioration. Hence, $\alpha$-P offers more biofriendly behaviors as compared to graphene and is expected to offer a variety of functions through the adsorption of these organic molecules. Additionally, to enhance the binding we also considered adsorption of these organic molecules to gold clusters attached to the surface of $\alpha$-P. Gold nanoparticles by themselves offer uniquely easy functionalization; they display colors depending on their size, shape, and state of aggregation, and they are known excellent tools in the field of nanobiotechnology and medicine. They are generally capped by organic molecules or amino acids to enhance structural stability and photo- luminescence characteristics. ${ }^{37-41}$ It is found that DNA molecules attached to $\mathrm{Au}$ nanoparticles acquire enhanced sensitivity and more capability for the hybridization process. ${ }^{42}$ $\mathrm{Au}$ nanoparticles allow also fast and direct electron transfer between a wide range of electroactive species and electrode materials. Additionally, light-scattering properties and extremely large enhancement ability of the local electromagnetic field enables Au nanoparticles to be used as signal amplification tags in diverse biosensors. ${ }^{43}$ In view of the fact that the binding of glycine was significantly enhanced on a template consisting of the defect patterned graphene to attain self-assembly behavior, ${ }^{44}$ we also explored the adsorption of one of the best known amino acids, that is, tyrosine at the vacancy and divacancy sites of $\alpha$-P.

Our study starts with the characterization of the amino acid and nucleobase molecules (glutamine, histidine, tyrosine, adenine, guanine, cytosine, and thymine) and the bare $\alpha$-P monolayer. Then, we determined the equilibrium binding configurations with highest binding energies and the corresponding electronic structures of seven different individual amino acids and nucleobases on bare $\alpha$-P based on an extensive search of possible binding geometries. To achieve relatively stronger binding interactions and hence to attain their fixation, we also explored their equilibrium binding configurations and energies to gold atom clusters $\left(\mathrm{Au}_{x}, x=1-\right.$ 5 ) and also a single Ti atom attached to the $\alpha$-P monolayer, as well as single-vacancy and divacancy sites in the $\alpha$-P monolayer. It should be noted that this exploration of the equilibrium binding structures to bare and adatom (cluster) and vacancy covered phosphorene monolayers involved extensive optimization studies. Critical effects of the coverage of bare $\alpha$-P monolayer with gold clusters and Ti adatom have been unveiled from this study. Amino acid and nucleobase molecules adsorbed to these adsorbates on the bare $\alpha$-P monolayer acquired strong binding and led to functionalization of the substrate. In addition to the changes of electronic structure, we examined the effect of amino acid or nucleobase coating on the optical properties, in particular the absorbance of the substrate.

Computational Details. Density functional theory calculations were performed using the VASP package ${ }^{45,46}$ within the generalized gradient approximation (GGA). Exchange-correlation potential ${ }^{47}$ is represented by Perdew-Burke-Ernzerhof (PBE) functionals with the PAW pseudopotentials. ${ }^{48,49}$ The van der Waals (vdW) interaction was included by D2-Grimme correction (DFT-D2) $)^{50}$ in all calculations. Plane-wave basis sets with the kinetic energy cutoff value of $600 \mathrm{eV}$ were employed. The k-point mesh of $3 \times 3 \times 1$ was used for the Brillouin zone (BZ) integration according to the MonkhorstPack method. ${ }^{51}$ The energy convergence criteria of the electronic self-consistency was taken as $10^{-5} \mathrm{eV}$ between two successive iterations. The optimized structures were obtained when Hellman-Feynman forces on each atom became smaller than $0.02 \mathrm{eV} / \AA$. In order to mimic the interaction between an isolated, individual amino acid or nucleobase and the $\alpha$-P monolayer, calculations were carried out using $(4 \times 5)$ supercells of $\alpha$-P, where the spacing between $\alpha$-P planes was taken to be $20 \AA$ to avoid interactions between images. Since PBE calculations underestimate the HOMO-LUMO gap of amino acid (AA) and nucleobase (NB) molecules, as well as the band gap of the bare $\alpha$-P, we applied Heyd-ScuseriaErnzerhof hybrid functional correction (HSE) ${ }^{52}$ to PBE results with a default (standard) mixing parameter of 0.25 . This way, 


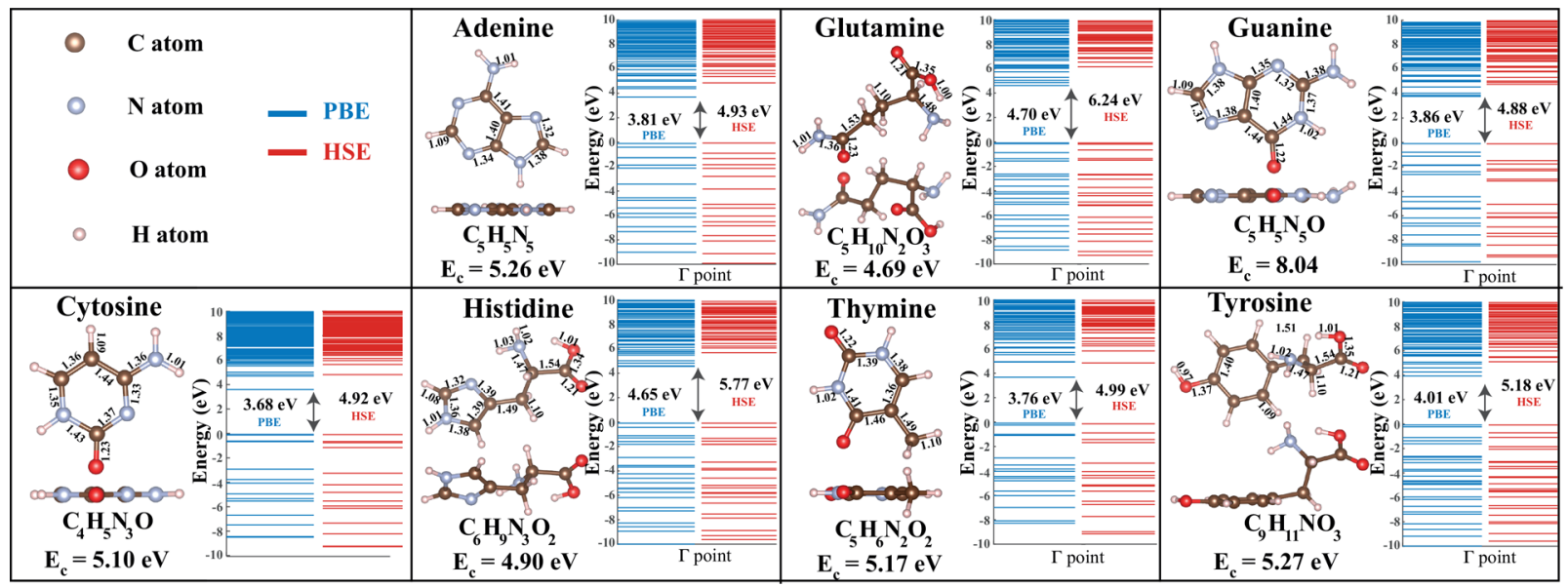

Figure 1. Optimized geometric structures with relevant bond lengths and electronic energy level structure with HOMO-LUMO gaps (HLGs) calculated by PBE and corrected by HSE of seven amino acid or nucleobase molecules. Calculated cohesive energies, $E_{\mathcal{c}}$ relative to free constituent atoms are also given.

the energy gap values significantly improved. However, owing to the nature of the screened Coulomb interaction approach, ${ }^{53}$ the HSE correction is not affordable for large systems comprising several atoms in the supercell of $\mathrm{AA} / \mathrm{NB}+\alpha-\mathrm{P}$ systems.

The binding energy, which measures the strength of the interaction between an amino acid or nucleobase and the $\alpha$-P monolayer was calculated from the following expression:

$$
E_{\mathrm{b}}=E_{\mathrm{T}}[\alpha-\mathrm{P}]+E_{\mathrm{T}}[\mathrm{AA} / \mathrm{NB}]-E_{\mathrm{T}}[\alpha-\mathrm{P}+\mathrm{AA} / \mathrm{NB}]
$$

in terms of the total energy of bare $\alpha$-P per supercell, $E_{\mathrm{T}}[\alpha-\mathrm{P}]$, of free amino acid or nucleobase, $E_{\mathrm{T}}[\mathrm{AA} / \mathrm{NB}]$, and of $\alpha-\mathrm{P}$ supercell having one $\mathrm{AA} / \mathrm{NB}$ adsorbed, $E_{\mathrm{T}}[\alpha-\mathrm{P}+\mathrm{AA} / \mathrm{NB}]$, respectively. Positive values of $E_{\mathrm{b}}$ indicate binding. The binding energy of free gold clusters, $\mathrm{Au}_{x}$, with $x=1-5$, per $\mathrm{Au}$ atom is calculated in terms of the total energy of a single $\mathrm{Au}$ atom and the total energy of a free gold cluster $\mathrm{Au}_{x}$, using the expression $E_{\mathrm{b}}=E_{\mathrm{T}}[\mathrm{Au}]-E_{\mathrm{T}}\left[\mathrm{Au}_{x}\right] / x$, where $\mathrm{AA} / \mathrm{NB}$ is replaced by $\mathrm{Au}_{x}$. The binding energy of a bare gold cluster to $\alpha$-P monolayer (per supercell) is calculated as $E_{\mathrm{b}}=E_{\mathrm{T}}\left[\mathrm{Au}_{x}\right]-E_{\mathrm{T}}\left[\mathrm{Au}_{x}+\alpha-\mathrm{P}\right]$. The binding energy of an AA or a NB to the gold cluster already adsorbed to the $\alpha-\mathrm{P}$ supercell is calculated as $E_{\mathrm{b}}=$ $E_{\mathrm{T}}\left[\alpha-\mathrm{P}+\mathrm{Au}_{x}\right]+E_{\mathrm{T}}[\mathrm{AA} / \mathrm{NB}]-E_{\mathrm{T}}\left[\alpha-\mathrm{P}+\mathrm{AA} / \mathrm{NB}+\mathrm{Au}_{x}\right]$, where $E_{\mathrm{T}}\left[\alpha-\mathrm{P}+\mathrm{AA} / \mathrm{NB}+\mathrm{Au}_{x}\right]$ is the total energy of an $\mathrm{AA}$ or a $\mathrm{NB}$ molecule adsorbed to a gold cluster that is already adsorbed to the $\alpha$-P supercell. The formation energies of vacancy defects $[\mathrm{D}]$ are calculated by the expression $E_{\mathrm{f}}=E_{\mathrm{T}}[\mathrm{D}]-E_{\mathrm{T}}[\alpha-P]+$ $n \mu[\mathrm{P}]$ in terms of the total energy of defect-containing $\alpha-\mathrm{P}$ having either a single vacancy or a divacancy with $n$ missing $\mathrm{P}$ atoms in the $\alpha$-P supercell, the total energy of the bare $\alpha-\mathrm{P}$ supercell and the atomic chemical potential, $\mu$, of a $\mathrm{P}$ atom. Here $n=1$ for a single vacancy per supercell and $n=2$ for a divacancy.

The analysis of the charge density and exchange of charge between $\mathrm{AA}$ or $\mathrm{NB}$ and $\alpha-\mathrm{P}$ is carried out by using the calculated total charge density, $\rho_{\mathrm{T}}$, and the difference charge density, $\Delta \rho=\rho_{\mathrm{T}}-\rho_{\mathrm{AA} / \mathrm{NB}}-\rho_{\alpha-\mathrm{P}}$, in terms of the charge density of free AA or NB molecules, $\rho_{\mathrm{AA} / \mathrm{NB}}$, and the charge density of the bare phosphorene monolayer, $\rho_{\alpha-\mathrm{P}}$. The nature of the bonding between an $\mathrm{AA}$ or a NB molecule and $\alpha$-P is clarified by this charge density analysis. Bader analysis is used for calculating the charge on atoms. ${ }^{54}$

\section{FREE AA OR NB MOLECULES AND BARE $\alpha$-P MONOLAYER}

In this section, we present a brief background about each AA or NB molecule concerning its significance and calculated values related to its atomic and electronic structure. For the sake of completeness, we also discuss the structural parameters, cohesive energy, and electronic band structure of the bare $\alpha$-P monolayer calculated with the same method as that for AA and NB molecules. Starting from the amino acids and nucleobases:

(i) Adenine is one of the nucleobases of both deoxyribonucleic acid (DNA) and ribonucleic acid (RNA) and has the chemical formula $\mathrm{C}_{5} \mathrm{H}_{5} \mathrm{~N}_{5}$. It is a planar molecule consisting of adjacent hexagonal and pentagonal rings with a HOMO-LUMO gap (HLG) calculated by $\mathrm{PBE}$ of $E_{\mathrm{HLG}}=$ $3.81 \mathrm{eV}$ as shown in Figure 1. The HLG of adenine increases to $4.93 \mathrm{eV}$ upon HSE correction. Adenine helps to stabilize the nucleic acid portion of DNA and RNA molecules. It is the fundamental component of adenosine triphosphate, which carries energy needed for all physiological processes in the cell.

(ii) Glutamine with its chemical formula $\mathrm{C}_{5} \mathrm{H}_{10} \mathrm{~N}_{2} \mathrm{O}_{3}$ does not constitute a ring structure. Its HOMO-LUMO gap is $E_{\mathrm{HLG}}=4.70 \mathrm{eV}$, which increases to $E_{\mathrm{HLG}}=6.24 \mathrm{eV}$ after HSE correction; it is widest among the AA molecules considered in this study. This AA molecule is a key ingredient necessary for anabolic growth of cells and provides nitrogen for protein and nucleotide synthesis. ${ }^{55}$

(iii) Guanine has a planar geometry consisting of one pentagonal ring adjacent to a hexagonal ring. It has chemical formula $\mathrm{C}_{5} \mathrm{H}_{5} \mathrm{~N}_{5} \mathrm{O}$ with $E_{\mathrm{HLG}}=3.86 \mathrm{eV}$, which increases to $E_{\mathrm{HLG}}=4.88 \mathrm{eV}$ after HSE correction. Guanine forms guanosine triphosphate, which mediates the transport of signals within the cell.

(iv) Cytosine is one of the four main bases found in DNA and RNA, along with adenine, guanine, and thymine (uracil in RNA). Cytosine is used in storing and transporting genetic information within a cell in the nucleic acids DNA and RNA like adenine and guanine. Its chemical formula is $\mathrm{C}_{4} \mathrm{H}_{5} \mathrm{~N}_{3} \mathrm{O}$, and it can pair with guanine. It forms cytidine triphosphate (CTP); it can act as a cofactor to enzymes and can transfer a phosphate to convert ATP.

(v) Histidine is one of the most essential AAs for growth of the human body and serves as a regulator of metal transmission 
a)

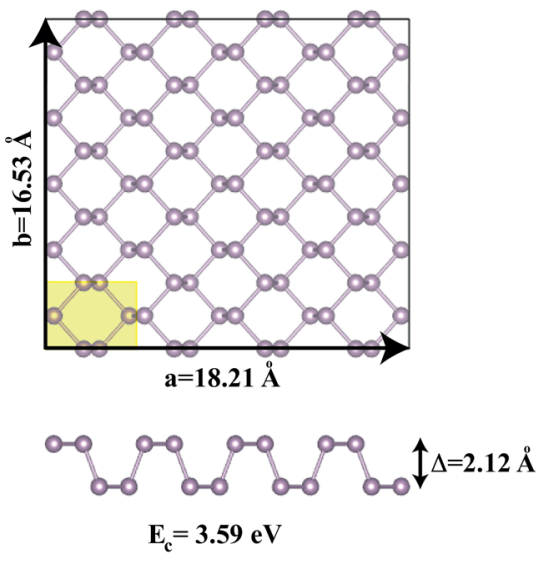

b)

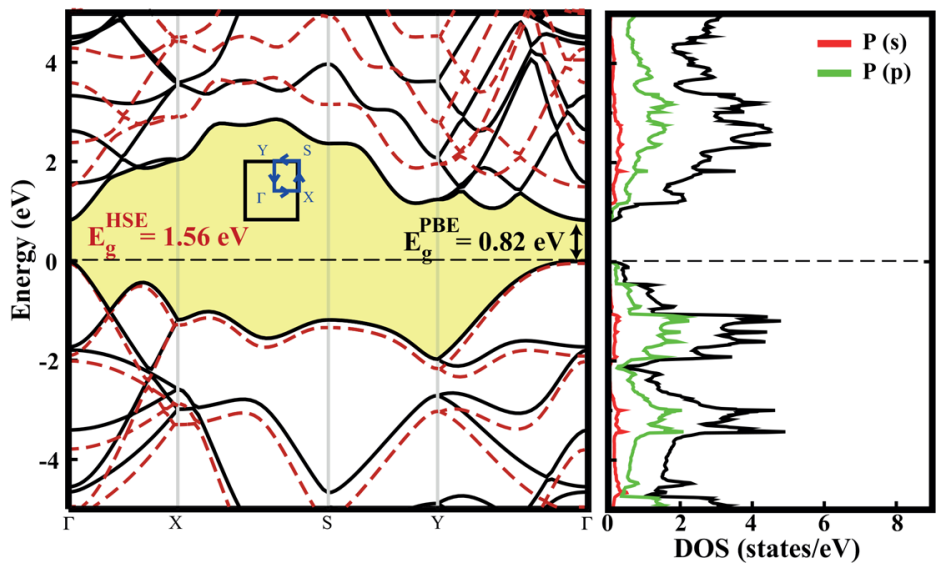

Figure 2. (a) Top and side views of the $(4 \times 5)$ supercell of $\alpha$-P (black phosphorene in symmetric washboard structure) used in the adsorption of the AA or NB molecules. The buckling and the cohesive energy per atom are also indicated. The rectangular primitive unit cell is shaded. (b) Energy band structure and the total and orbital projected partial densities of states calculated by PBE. Bands at the edges of conduction and valence bands corrected by HSE are shown by dashed lines.

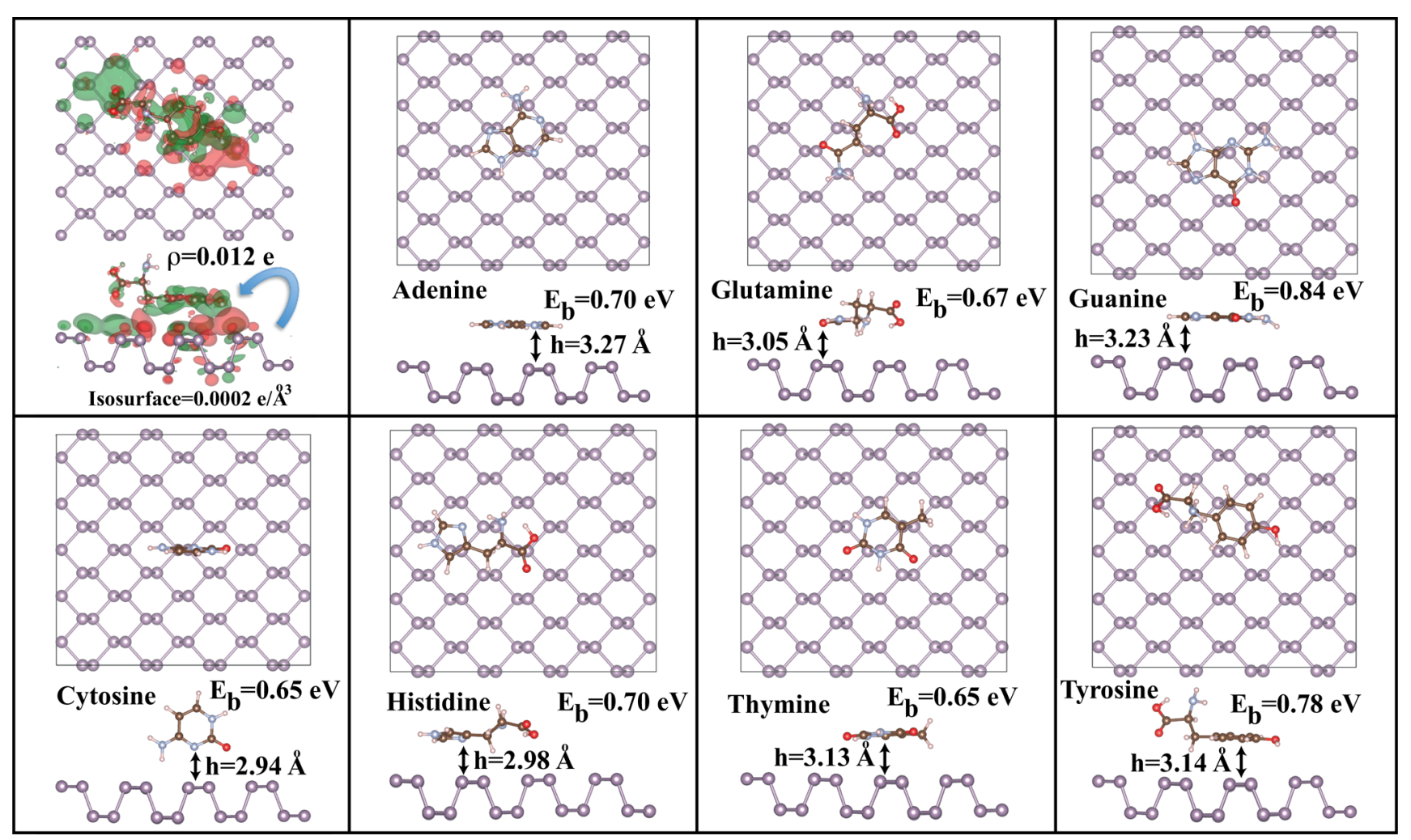

Figure 3. (top, left) Top and side views of the isosurfaces of the difference charge density, $\Delta \rho(r)$, of a tyrosine molecule physisorbed to an $\alpha$-P monolayer. Charge depletion and charge accumulation zones are shown by green and light red colors, respectively. $\rho$ is the charge transfer from $\alpha$-P to tyrosine. Other panels show top and side views of equilibrium binding structures and binding energies, $E_{\mathrm{b}}$, of amino acid and nucleobase molecules on the $\alpha$-P monolayer. The physisorption of individual molecules on $\alpha$-P is mimicked within the supercell geometry, where one molecule physisorbed to each supercell is periodically repeated. The lattice constants of the $(4 \times 5)$ supercell are $a=18.21 \AA, b=16.53 \AA$, and $c=20 \AA$.

in biological systems. It has only one pentagonal ring with chemical formula $\mathrm{C}_{6} \mathrm{H}_{9} \mathrm{~N}_{3} \mathrm{O}_{2}$. Its PBE calculated HLG, $E_{\mathrm{HLG}}=$ $4.65 \mathrm{eV}$, increases to $5.77 \mathrm{eV}$ after HSE correction.

(vi) Thymine with chemical formula $\mathrm{C}_{5} \mathrm{H}_{6} \mathrm{~N}_{2} \mathrm{O}_{2}$ is one of four nucleobases that form the basic building blocks of DNA and consists of one single hexagonal ring. Its gap, $E_{\mathrm{HLG}}=3.76$ $\mathrm{eV}$, increases to $4.99 \mathrm{eV}$ after HSE correction. (vii) Finally, tyrosine is a vital constituent of protein. Its chemical formula $\mathrm{C}_{9} \mathrm{H}_{11} \mathrm{NO}_{3}$ has only one hexagonal ring. The gap is calculated to be $E_{\mathrm{HLG}}=4.01 \mathrm{eV}$, which increases to 5.18 eV after HSE correction.

The black phosphorene monolayer, which is used as a substrate to fix AA and NB molecules, is stable in suspended 2D symmetric washboard structure and is obtained from black phosphorus. It has a rectangular unit cell with four $\mathrm{P}$ atoms, 


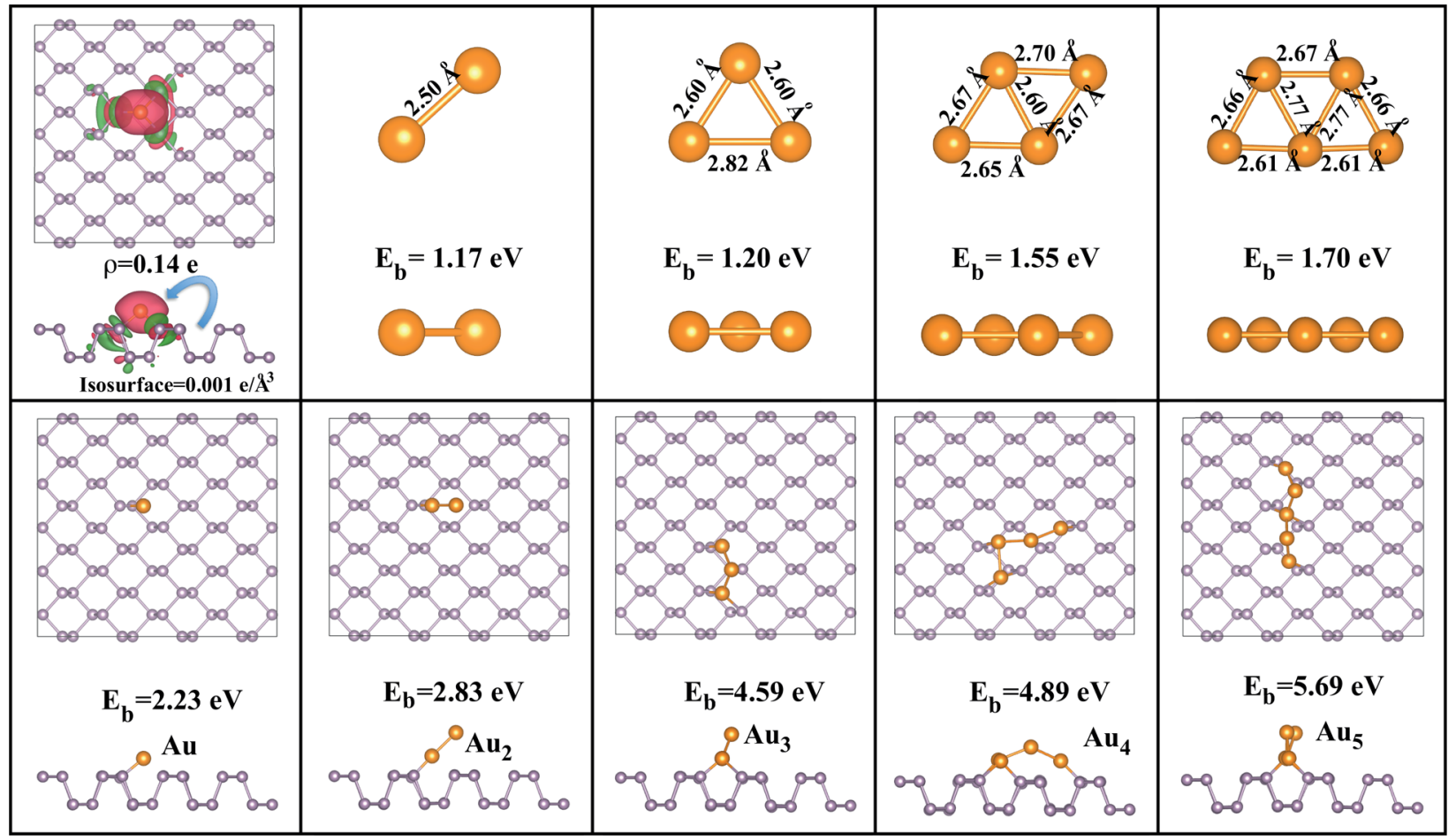

Figure 4. (top, left) Top and side views of isosurfaces of the difference charge density, $\Delta \rho(r)$, of a single Au atom adsorbed to the $\alpha$-P monolayer. Charge depletion and charge accumulation zones are shown by green and light red colors, respectively. Other top row panels show top and side views of optimized structures of free gold $\mathrm{Au}_{x}$ with $x=1-5$ clusters and their binding energy per atom. Bottom row panels show top and side views of the optimized atomic structure of gold clusters adsorbed to the $\alpha$-P monolayer and calculated binding energies per cluster, $E_{\mathrm{b}}$.

and a direct fundamental band gap of $E_{\mathrm{g}}=0.82 \mathrm{eV}$, which is increased to $1.56 \mathrm{eV}$ upon the HSE correction. These values are comparable to the values predicted in previous studies of $0.90 \mathrm{eV}^{56,57}$ and $1.66 \mathrm{eV}$ respectively, ${ }^{56}$ but still $0.64 \mathrm{eV}$ smaller than the experimental value. Nonetheless, one can attain the measured value of $E_{\mathrm{g}}=2.23 \mathrm{eV}$ using HSE correction with a mixing parameter of 0.5 . However, this would be a fitting to an experimental energy gap, rather than the prediction of it. We note that the underestimation of HLG of $\mathrm{AA}$ and NB molecules or the energy band gap of $\alpha$-P have only a minute effect on the strength of the binding, which is a ground state property. Also, the binding interaction of an AA or a NB molecule with the $\alpha$-P monolayer is predominantly weak vdW attraction, which is not affected significantly by the underestimated excited states.

Few-layer black phosphorene field effect transistors $(\text { FETs })^{58,59}$ have a high field-effect mobility up to $286 \mathrm{~cm}^{2} /$ $(\mathrm{V} \mathrm{s})$ at room temperature. Transport properties like carrier mobilities $^{60,61}$ and thermal conductivity ${ }^{62,63}$ have been reported to be highly anisotropic on a $\alpha$-P monolayer. As compared to graphene, organic molecules on $\alpha$-P undergo relatively less deterioration. Additionally, $\alpha-\mathrm{P}$ is accepted as a template for the self-assembly of DNA/RNA constituents and is better than graphene. These properties together with favorable electronic properties of $\alpha$-P summarized above make the $\alpha$-P monolayer superior to graphene in biological applications. In Figure 2 optimized structural parameters, energy band structure, and corresponding density of states of the $\alpha$-P monolayer are presented for the sake of completeness.

\section{BINDING OF AA AND NB MOLECULES TO BARE $\alpha$-P MONOLAYER}

The equilibrium configuration of an amino acid or a nucleobase molecule attached to a bare $\alpha$-P monolayer is determined using a supercell geometry. Following the full relaxations of all free (isolated) AA or NB molecules and of the free, bare $\alpha$-P monolayer, each AA or NB molecule is initially placed above specific sites of the $(4 \times 5)$ supercell (see Figure 2 a), over $\mathrm{C}, \mathrm{N}$, and $\mathrm{O}$ ends, which is periodically repeated. Then, the atomic structure of the AA or NB molecule $-\alpha-\mathrm{P}$ monolayer system is further optimized within the supercell geometry. Among all binding configurations taken into account, the maximum binding energy (or minimum total energy) configuration is considered to be an equilibrium geometry. The binding of an individual (or isolated) AA or NB molecule on bare $\alpha$-P monolayer is ensured by taking large supercell size $(a=18.21 \AA, b=16.53 \AA$, and $c=20 \AA)$ and large spacing $(c=20 \AA)$ between periodically stacked monolayers, whereby the coupling between adjacent AA or NB molecules is minimized.

Upon relaxation of all atoms in the system, most of the molecules preferred to orient themselves parallel to the monolayer, as shown in Figure 3 . The binding energies, $E_{\mathrm{b}}$, of these molecules on $\alpha$-P are calculated to be in the range of $0.65-0.84 \mathrm{eV}$. The binding energies of some AA molecules calculated in earlier studies ${ }^{4,64}$ are close to the values predicted in this study. These energies indicate that the binding is stronger than pure vdW interaction and includes small chemical interactions by minute charge exchange between the AA or NB molecule and $\alpha$-P. Accordingly, small variations of predicted binding energies among $\mathrm{AA}$ and $\mathrm{NB}$ molecules treated here are attributed to the differences in their sizes and 

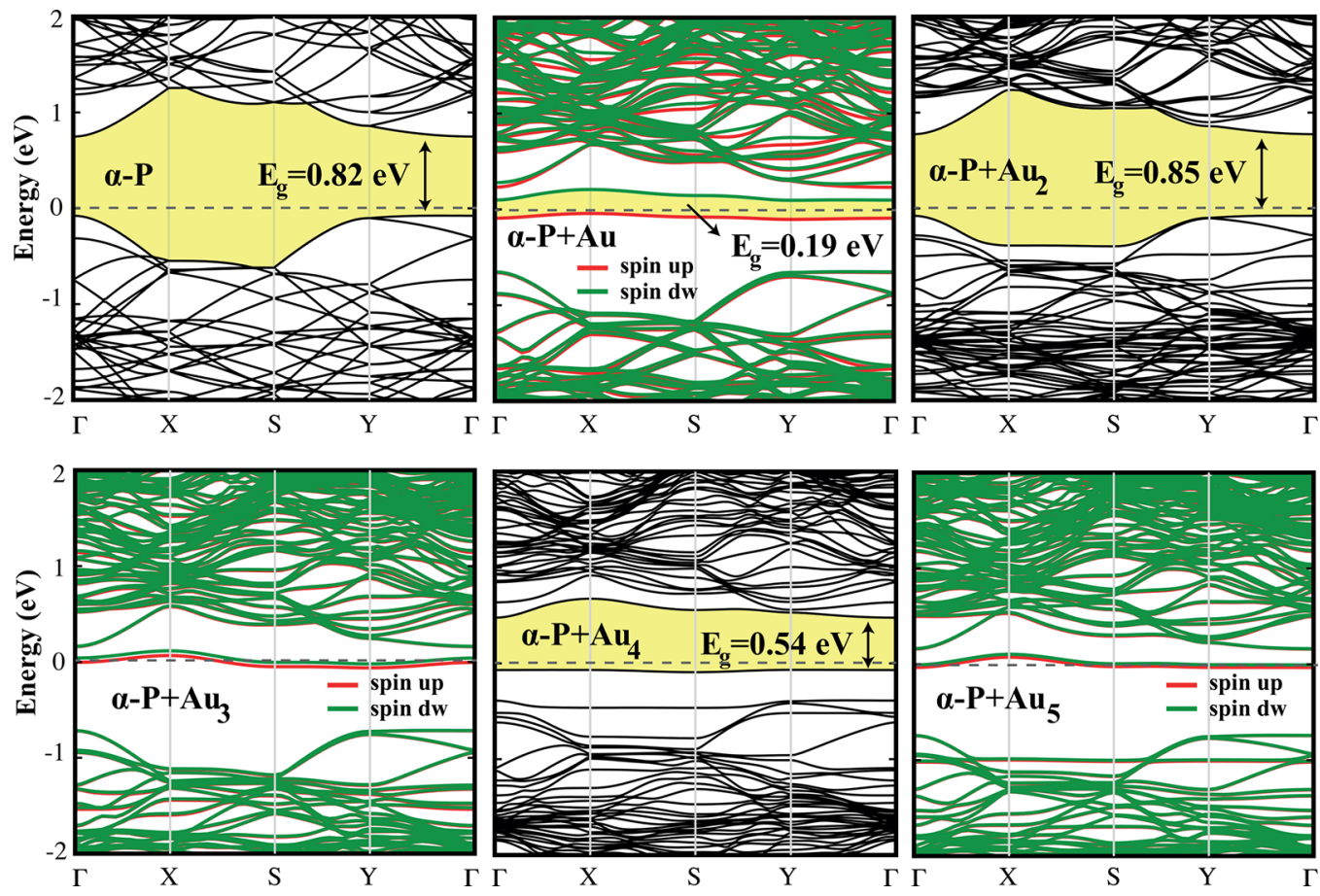

Figure 5. Electronic energy band structures of gold clusters, $\mathrm{Au}_{x}(x=1-5)$ adsorbed to the bare $\alpha$-P monolayer are calculated within the $(4 \times 5)$ supercell geometry.

atomic structures and equilibrium binding geometries. Isosurfaces of the difference charge density, $\Delta \rho(r)$, and the charge transfer of $\rho=0.012 \mathrm{e}$ from $\alpha$-P to AA molecule calculated for tyrosine $+\alpha$-P monolayer in the first panel of Figure 3 describes the character of the binding, which is typical for the amino acid molecules we studied here. As a consequence of the weak binding, no significant changes in the energy band structure of $\mathrm{AA} / \mathrm{NB}+\alpha-\mathrm{P}$ monolayer are seen in Figure $\mathrm{S} 1$.

\section{BINDING OF AA AND NB MOLECULES TO GOLD CLUSTER COADSORBED TO $\alpha$-P MONOLAYER}

The binding energy and hence the fixation of an AA or a NB molecule can be enhanced by binding at a defect site, like a vacancy, ${ }^{44}$ or adatom or cluster. Motivated by earlier works finding gold to be a potential element in biosensor applications of graphene, ${ }^{65-67}$ we consider first gold clusters on the $\alpha$-P monolayer as adspecies to promote the binding of $\mathrm{AA}$ or $\mathrm{NB}$ molecules. To this end, we first determined the structure of optimized free gold clusters, $\mathrm{Au}_{x}$ with $x=1-5$, and their binding energies per atom in Figure 4. These equilibrium structures are planar and consistent with earlier studies. ${ }^{68,69}$ Gold clusters with odd numbers of $\mathrm{Au}$ atoms acquire also a permanent magnetic moment of $1.0 \mu_{\mathrm{B}}$. Then, the equilibrium geometries of the gold clusters on the $\alpha$-P monolayer were determined by placing a single Au atom on possible adsorption sites, such as hollow site, top site, bridge site, etc. Using this information we next investigated the adsorption of other nanoclusters, $\mathrm{Au}_{x}$ with $x=1-5$, to $\alpha$-P; their equilibrium configurations were determined following a structure optimization process by testing all possible adsorption sites. The equilibrium atomic structures of these gold clusters adsorbed to the $\alpha$-P monolayer are also shown in Figure 4. Notably, in spite of the fact that the ground state optimal structures of these gold clusters are planar (up to $x=7$ ), ${ }^{69}$ some of them underwent structural changes after the adsorption to the $\alpha-\mathrm{P}$ monolayer. For example, the free $\mathrm{Au}_{3}$ cluster has an equilibrium structure like a isosceles triangle shape with a base of $2.82 \AA$, which increases to $3.58 \AA$ upon the adsorption of the $\mathrm{Au}_{3}$ cluster on the $\alpha$-P monolayer. While a free $\mathrm{Au}_{4}$ cluster has a rhomboid shape, it undergoes a significant structural modification upon adsorption to the $\alpha$-P monolayer and attains the shape of a zigzag chain. Similarly, the free $\mathrm{Au}_{5}$ cluster has a symmetric shape like two collateral triangles, in other words it has an extra atom next to the rhomboid structure. However, it is stretched out like a zigzag chain after relaxation on the $\alpha$-P monolayer. Briefly, free $\mathrm{Au}_{x}$ clusters in equilibrium are transformed to generate zigzag chains on the $\alpha$ $\mathrm{P}$ monolayer.

The binding energy of an adsorbed single $\mathrm{Au}$ atom, $E_{\mathrm{b}}=$ $2.23 \mathrm{eV}$, is rather high and indicates the usual chemical bond pertaining to chemisorption. The binding energy of clusters to $\alpha$-P monolayer increases with increasing number of $\mathrm{Au}$ atoms in the cluster, which form a few chemical bonds with the underlying $\mathrm{P}$ atoms of the substrate. According to the Bader charge analysis, electronic charge tends to transfer from the bare $\alpha$-P monolayer to the $\mathrm{Au}_{x}$ cluster. This behavior is in compliance with the electronegativity of $\mathrm{Au}\left(\Xi_{\mathrm{Au}}=2.4\right)$, which is higher than that of $\mathrm{P}\left(\Xi_{\mathrm{P}}=2.1\right)$. The amount of charge transferred to the adsorbed $\mathrm{Au}$ cluster increases with the increasing number of $\mathrm{Au}$ atoms in the range of 0.14-0.66 electrons. In addition, we performed also an analysis based on orbital projected density of states to reveal the bonding mechanism of $\mathrm{Au}_{x}$ clusters on the $\alpha$-P monolayer as shown in Figure S2. It is clear that contributions around Fermi level are mainly due to Au s-orbital. Moreover sp-hybridization can be arise from overlapping of $\mathrm{Au} \mathrm{s}$ - and $\mathrm{P}$ p-orbitals in the same energy ranges.

The single $\mathrm{Au}$ atom adsorbed to the $\alpha$-P monolayer is spin polarized with a magnetic moment of $\mu=1 \mu_{\mathrm{B}}$. In the case of an individual isolated $\mathrm{Au}$ impurity, two magnetic impurity 


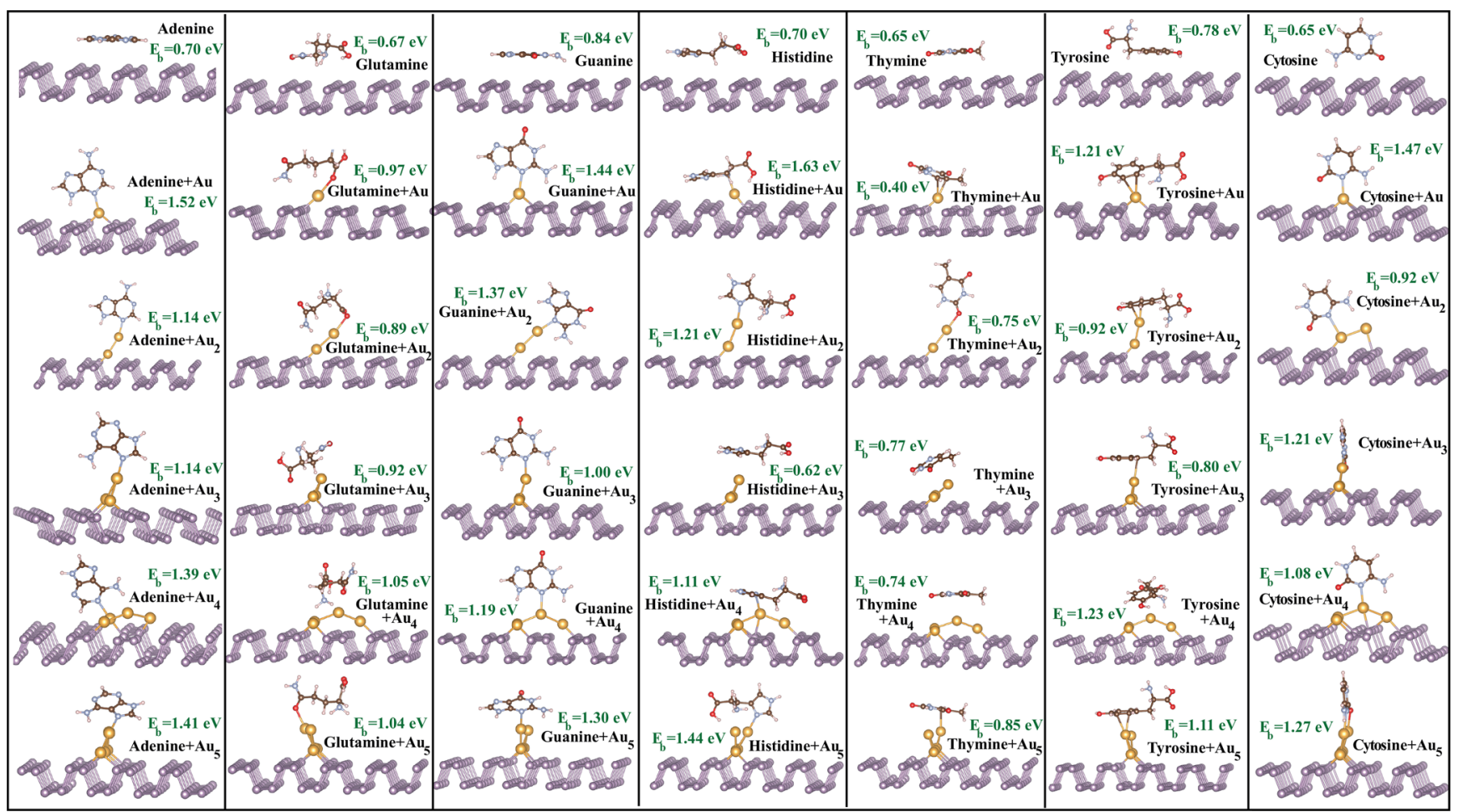

Figure 6. Perspective view of optimized atomic structures of AA and NB molecules adsorbed to $\alpha$-P (first row) and to the gold clusters (Au with $x$ $=1-5)$, which were already adsorbed to $\alpha$-P monolayer. The binding energies of AA and NB molecules to gold clusters are also given. Each equilibrium configuration presented here is determined by optimizing a number of possible adsorption geometries to find the lowest energy configuration.

localized states, one fully occupied above the midgap and one empty spin polarized one, are introduced. In the band pictures corresponding to uniform coverage of Au atom, these localized states construct one occupied and one empty spin polarized, flat band in the band gap and open a small band gap of 0.19 $\mathrm{eV}$. This way, the band gap of the bare $\alpha$-P monolayer can be modified depending on the decoration of adsorbed $\mathrm{Au}$ atoms. The clusters $\mathrm{Au}_{3}$ and $\mathrm{Au}_{5}$ have minute magnetic moments due to small spin-split of Au localized states at the Fermi level and are in metallic state. The clusters with even numbers of $\mathrm{Au}$ atoms maintain the semiconducting property of $\alpha$-P. Incidentally, while $\mathrm{Au}_{2}+\alpha$-P is a semiconductor with a band gap of $0.85 \mathrm{eV}$, the band gap of $\mathrm{Au}_{4}+\alpha-\mathrm{P}$ is reduced to 0.54 $\mathrm{eV}$. As seen, one can attain interesting functionalization of the $\alpha$-P monolayer through its decorations with single Au atoms or clusters of diverse sizes. For example, dramatic changes of the energy band structure of $\alpha-\mathrm{P}$ as a result of its decoration with $\mathrm{Au}_{x}(x=1-5)$ within the supercell geometry are depicted in Figure 5 .

We next analyze the interaction between $\mathrm{Au}_{x}$ clusters adsorbed to $\alpha$-P and AA or NB molecules. The determination of the equilibrium binding structure and related binding energy for each $\mathrm{AA}$ or $\mathrm{NB}$ molecule in different Au clusters required the optimization of several configurations starting from diverse possible initial configurations and is rather tedious. We considered different types of end atoms in each AA or NB molecule and placed the AA or NB molecule on $\mathrm{Au}_{x}$ clusters in parallel and vertical configuration over these different types of end atoms. For example, adenine has three types of atoms, carbon, nitrogen, and hydrogen. We placed the adenine molecule on the $\mathrm{Au}_{x}$ adsorbed to $\alpha$-P in parallel and vertical configuration with its carbon, nitrogen, and hydrogen atoms being close to the cluster. In this way, we determined the minimum energy configuration for each $\mathrm{AA}$ or $\mathrm{NB}$ adsorbed to the $\mathrm{Au}_{x}$ cluster after extensive structure optimizations of these several initial positions. Upon checking the binding energy values of these different adsorption geometries of adenine, the difference of the binding energies between the most stable and other binding configurations to $\mathrm{Au}_{1}+\alpha$-P range between 0.1 and $0.7 \mathrm{eV}$. This difference is about $0.4 \mathrm{eV}$ for $\mathrm{Au}_{2}+\alpha-\mathrm{P}$ substrate. The binding energy of the most stable configuration for adenine adsorbed to $\mathrm{Au}_{3}+\alpha$-P substrate is about $0.4 \mathrm{eV}$ higher than the other adsorption configurations. This difference ranges between 0.15 and $0.51 \mathrm{eV}$ for $\mathrm{Au}_{4}+\alpha$-P substrate. Similarly, the energy difference between the most stable configuration and the other relatively smaller energy configurations for adenine adsorbed to $\mathrm{Au}_{5}+\alpha$-P substrate is about $0.15 \mathrm{eV}$. We obtained also energy differences for the other $\mathrm{AA}$ or $\mathrm{NB}$ molecule $+\alpha$-P systems like those of adenine. In Figure 6, we just present the results for 35 equilibrium states and their binding energies obtained after the optimization of a large number of different possible adsorption configurations for $\mathrm{AA} / \mathrm{NB}+\mathrm{Au}_{x}+\alpha-\mathrm{P}$ with $x=1-5$. The calculated binding energies of $\mathrm{AA}$ or $\mathrm{NB}$ molecules to various Au clusters are generally higher than those to bare $\alpha$-P monolayer; but they change depending on the type of the molecule and Au cluster. It should be noted that each $\mathrm{Au}_{x}+\alpha$-P system has different selectivity toward $\mathrm{AA}$ and $\mathrm{NB}$ molecules due to increasing number of $\mathrm{Au}$ atoms. Also, the variation of binding of AA or NB molecules with the size of $\mathrm{Au}$ clusters is attributed to change of the active sites of $\mathrm{Au}_{x}$ clusters. $E_{\mathrm{b}}$ as high as $1.63 \mathrm{eV}$ indicates a rather strong chemical bond formed between an AA or a $\mathrm{NB}$ molecule and $\mathrm{Au}$ atom. Except for the thymine molecule, which forms weak bonds with all gold clusters, the 
a)

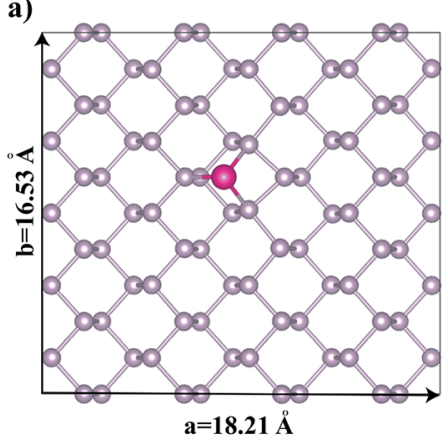

b)

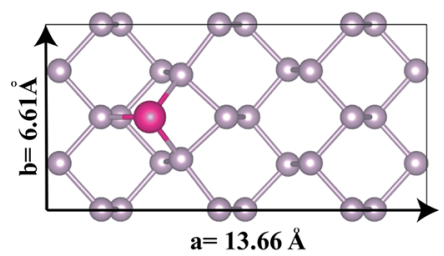

c)

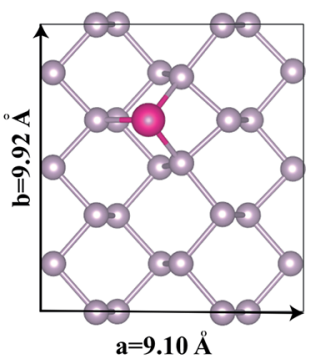

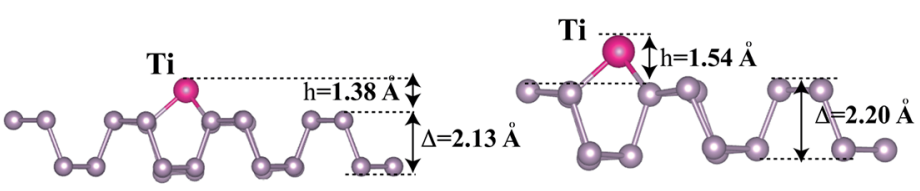
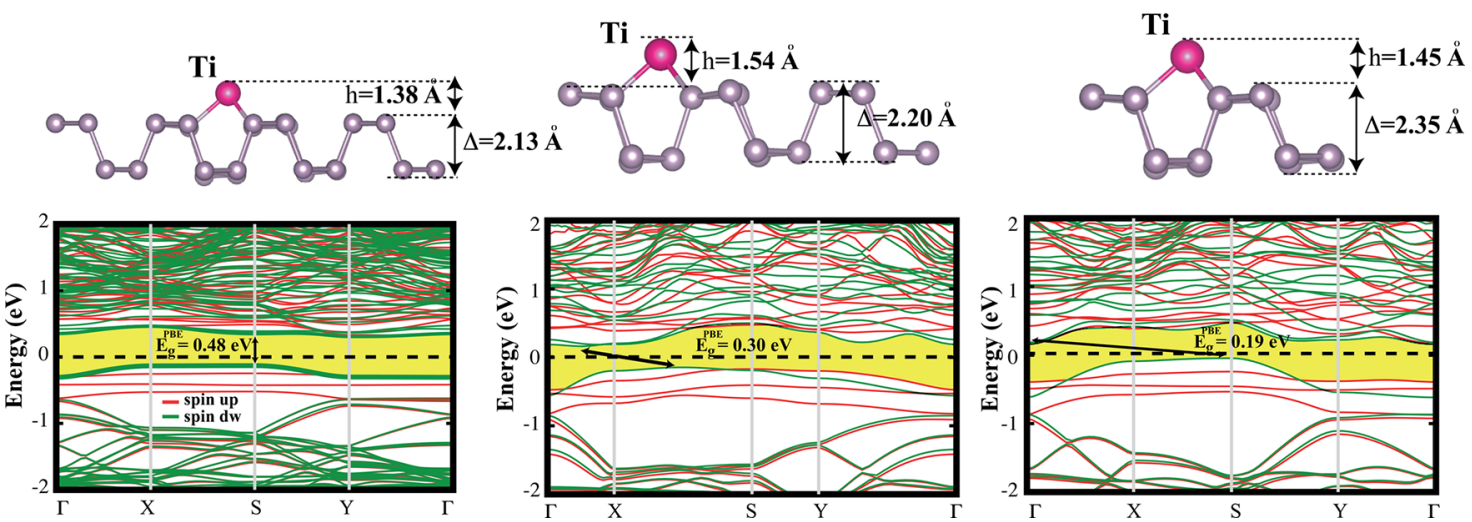

Figure 7. Equilibrium atomic configuration of Ti adatom in different patterns on the $\alpha$-P monolayer and corresponding energy band structures: (a) $(4 \times 5),(b)(3 \times 2)$, and $(c)(2 \times 3)$ patterns of the adsorbed Ti adatom.

binding and hence the fixation of AA or NB molecules to the $\alpha$-P monolayer can be enhanced by the coadsorption of $\mathrm{Au}$ clusters. Based on this result, one can argue that the selfassembly of various $\mathrm{AA}$ and $\mathrm{NB}$ molecules on $\alpha$-P can be achieved through the decoration of the $\alpha$-P monolayer by $\mathrm{Au}$ atoms or by specific Au clusters. This way the ordered coating of $\alpha$-P by organic molecules can be realized.

While the electronic structure of bare $\alpha$-P monolayer was not affected significantly after the adsorption of $\mathrm{AA}$ or $\mathrm{NB}$ molecules (as depicted by DOS and PDOS of $\mathrm{AA} / \mathrm{NB}+\alpha-\mathrm{P}$ given in Figure $S 1$ ), the situation is rather different in the case of the adsorption of $\mathrm{AA}$ or $\mathrm{NB}$ molecules to gold clusters already adsorbed to $\alpha$-P monolayers. The AA and NB molecules, which form strong chemical bonds with the gold clusters, give rise to changes in the electronic structure of the $\mathrm{Au}_{x}+\alpha$-P system. Our analysis of the atom projected PDOS of $\mathrm{AA} / \mathrm{NB}+\mathrm{Au}_{x}+\alpha$-P comprise a large number of data, which can be retrieved from Figure S3 in Supporting Information. We first explain the context of the analysis based on the PDOSs in Figure S3 and give a few relevant examples. Here, the PDOS of the $\mathrm{P}$ atom that is farthest from the $\mathrm{AA} / \mathrm{NB}+$ gold cluster complex on $\alpha$-P gives the DOS of the bare $\alpha$-P monolayer. This way the shift of the Fermi level and all the relevant gap states relative to the band gaps of the bare $\alpha$-P monolayer are taken into account. Accordingly, the states projected to the $\mathrm{AA} / \mathrm{NB}$ + gold cluster complex, which are within the band gap of the $\mathrm{P}$ host atom farthest from the complex, are the localized impurity states due to the absorbate in low coverage or dilute chemical doping limit. This information is crucial in determining the positions of impurity states in dilute chemical doping as mimicked in this study. In Figure S3, for example, the tyrosine molecule adsorbed to the $\mathrm{Au}_{4}$ cluster has two localized gap states derived from gold located below the Fermi level, whereas the states derived from this amino acid occurs as resonances in the valence band. We present the following conclusions retrieved from the analysis of PDOS presented in Figure S3:

The energy states are shifted toward lower energies after the adsorption of the AA or NB molecule. Some of the impurity states in the band gap disappear after the adsorption of these molecules. Moreover, the magnetic state of the $\mathrm{Au}+\alpha-\mathrm{P}$ system with a magnetic moment of $1.0 \mu_{\mathrm{B}}$ disappears. The adsorption of an $\mathrm{AA}$ or a NB molecule to the $\mathrm{Au}_{2}+\alpha$-P system maintains semiconductor behavior and does not show any impurity state in the band gap. The band gap values of adenine $(0.85 \mathrm{eV})$, glutamine $(0.83 \mathrm{eV})$, guanine $(0.84 \mathrm{eV})$, histidine $(0.80 \mathrm{eV})$, thymine $(0.83 \mathrm{eV})$, tyrosine $(0.88 \mathrm{eV})$, and cytosine $(0.78 \mathrm{eV})$ slightly change from the bare $\alpha$-P band gap value. The DOS of glutamine and tyrosine adsorbed to $\mathrm{Au}+\alpha-\mathrm{P}$ show similar trends except their intensity. Interestingly, the thymine $+\mathrm{Au}_{3}+\alpha$-P system is magnetic with $\mu=0.74 \mu_{\mathrm{B}}$ per cell. Notably, characteristic energy states of the AA or NB molecule can be observed in the DOS of $\mathrm{AA} / \mathrm{NB}+\mathrm{Au}_{x}+\alpha$-P projected to the AA or NB molecule.

Here we place an emphasis on the adsorption of the tyrosine molecule to gold clusters on the $\alpha$-P monolayer, because of the importance of phosphorylation mechanism by tyrosine kinase. Tyrosine prefers to be adsorbed to $\mathrm{Au}_{x}+\alpha$-P substrate over the $\mathrm{C}$ or $\mathrm{H}$ atom depending on the size of the coadsorbed gold cluster. Accordingly it displays rather different adsorption geometries depending on the size of the Au cluster, where the binding energies range from 0.80 to $1.23 \mathrm{eV}$. While the binding of tyrosine changes with the size of $\mathrm{Au}$ cluster, the electronic structure of tyrosine $+\mathrm{Au}_{x}+\alpha$-P monolayer shows dramatic change for $x=1$ as shown in Figure S4. According to the Bader analysis, a charge of $\sim 0.3$ electrons is back-donated to the $\alpha$-P monolayer from $\mathrm{Au}$ and tyrosine. At the end, while a single $\mathrm{Au}$ atom adsorbed to $\alpha$-P can be a magnetic, narrow band gap 
semiconductor, the binding of tyrosine to this coadsorbed $\mathrm{Au}$ atom results in a metallic state. The effect of adsorbed tyrosine on the electronic structure of $\mathrm{Au}_{x}+\alpha$-P is, however, minute for $x=2-5$, since chemisorption states occur below the top of the valence band gap of $\mathrm{Au}_{x}+\alpha$-P.

\section{BINDING OF AN AA MOLECULE TO TITANIUM ADATOM ON $\alpha$-P}

Coadsorption of AA molecules with a $3 \mathrm{~d}$ transition metal atom such as $\mathrm{Ti}$ is another aspect that we clarify in this work. Here we start with the analysis of the binding of free $\mathrm{Ti}$ atom to the bare $\alpha$-P monolayer. Similar to other washboard structures, ${ }^{70,71}$ $\mathrm{Ti}$ is adsorbed close to the hollow site of the $\alpha$-P monolayer. The binding energy, $E_{\mathrm{b}}=3.92 \mathrm{eV}$, indicates a strong chemical bonding with $\alpha$-P. Three bonds between adsorbed Ti and $\alpha$-P can occur with distances ranging from 2.37 to $2.44 \AA$ to nearest host $\mathrm{P}$ atoms. These bond distances are consistent with the results in literature. ${ }^{72,73}$ The height of the Ti atom from the $\alpha$ $\mathrm{P}$ plane is $1.38 \AA$ as shown in Figure 7. Upon one $\mathrm{Ti}$ adsorption to each $(4 \times 5)$ supercell, the nonmagnetic bare $\alpha$ $\mathrm{P}$ monolayer attains a magnetic moment of $2.0 \mu_{\mathrm{B}}$ per supercell, and its direct band gap decreases from 0.82 to $0.48 \mathrm{eV}$. We found that the electronic structure, in particular, the width and type of the band gap, is strongly dependent on the coverage and decoration of the $\mathrm{Ti}$ adatom. For example, for $(3 \times 2) \mathrm{Ti}$ coverage, the direct band gap changes to indirect and is reduced to $0.30 \mathrm{eV}$, whereas the $(2 \times 3)$ coverage leads to the further reduction of the indirect band gap to be $0.19 \mathrm{eV}$. We believe that this is a crucial effect in the functionalization of bare $\alpha$-P monolayers.

Here, we consider only the binding of tyrosine to the $\mathrm{Ti}$ adatom adsorbed the to $\alpha$-P monolayer, that is, $\mathrm{Ti}+\alpha-\mathrm{P}$, for reasons explained above and calculate their optimized structures. The equilibrium structure with highest binding energy among all different binding configurations is shown in Figure 8. The binding energy is calculated to be $E_{\mathrm{b}}=2.44 \mathrm{eV}$. Apparently, the interaction between tyrosine and the $\alpha$-P monolayer and hence its binding is significantly enhanced through coadsorbed $\mathrm{Ti}$. The height of tyrosine from $\mathrm{Ti}$ and the $\alpha$-P plane is 1.33 and $2.67 \AA$, respectively. While the tyrosine + $\mathrm{Ti}+\alpha$-P system retains the magnetic moment value of $2.0 \mu_{\mathrm{B}}$ per supercell even after tyrosine adsorption, its direct band gap decreases from 0.48 to $0.16 \mathrm{eV}$.

\section{BINDING OF AN AA MOLECULE TO DEFECT SITES}

Since unsaturated atoms at the defect sites, like a single vacancy or divacancy, can be active sites and can form strong bonds with AA molecules, ${ }^{44}$ we consider first single vacancy and divacancy of the $\alpha$-P monolayer. A single vacancy is formed by removing one $\mathrm{P}$ atom from the monolayer. After the structure optimization the surrounding $\mathrm{P}$ atoms slightly displaced toward the missing $\mathrm{P}$ atom. The formation energy is calculated to be $1.96 \mathrm{eV}$. At the end, defect states occurred close to the edge of the valence band as shown in Figure 9. In the band picture within the supercell, the semiconducting bare $\alpha$-P monolayer is transformed to a metal in the presence of patterned vacancy. In contrast to the expectations, the binding of tyrosine at the vacancy occurred rather weakly, since tyrosine remained parallel to the monolayer and hence did not participate in forming a strong bond with one of the host $\mathrm{P}$ atoms surrounding the vacancy. Because of this weak vdW interaction between the vacancy patterned $\alpha$-P monolayer and
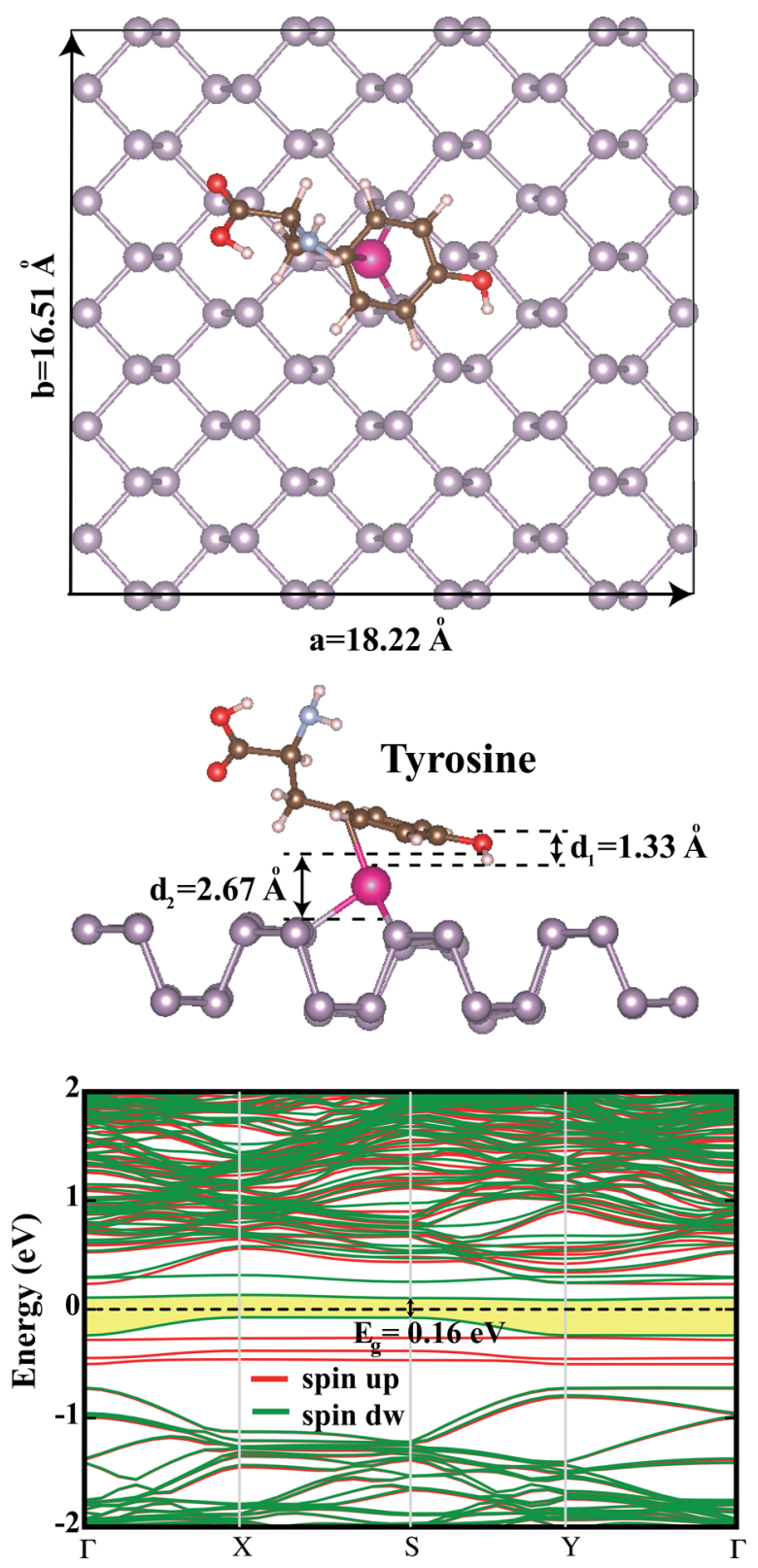

Figure 8. Optimized atomic structure of tyrosine adsorbed to $\mathrm{Ti}+\alpha$ $\mathrm{P}$ system according to the $(4 \times 5)$ supercell geometry and the corresponding electronic energy band structure. The band gap is shaded.

tyrosine, the band structure remained practically unaltered upon the physisorption (See Figure S5).

The formation energy of divacancy is not twice the formation energy of the single vacancy and is calculated to be $3.08 \mathrm{eV}$. In the patterned divacancy created in the $\alpha-\mathrm{P}$ monolayer, the band gap of bare $\alpha$-P monolayer is narrowed to $0.44 \mathrm{eV}$ as shown in Figure 9. Similar to the case of single vacancy, tyrosine, preferring the configuration parallel to the monolayer, did not form strong bonds with the unsaturated active $\mathrm{P}$ atoms surrounding the divacancy. In the end, binding to $\alpha$-P at the divacancy site is realized through weak vdW interaction, and the band structure of divacancy patterned $\alpha$-P monolayer did not change. 


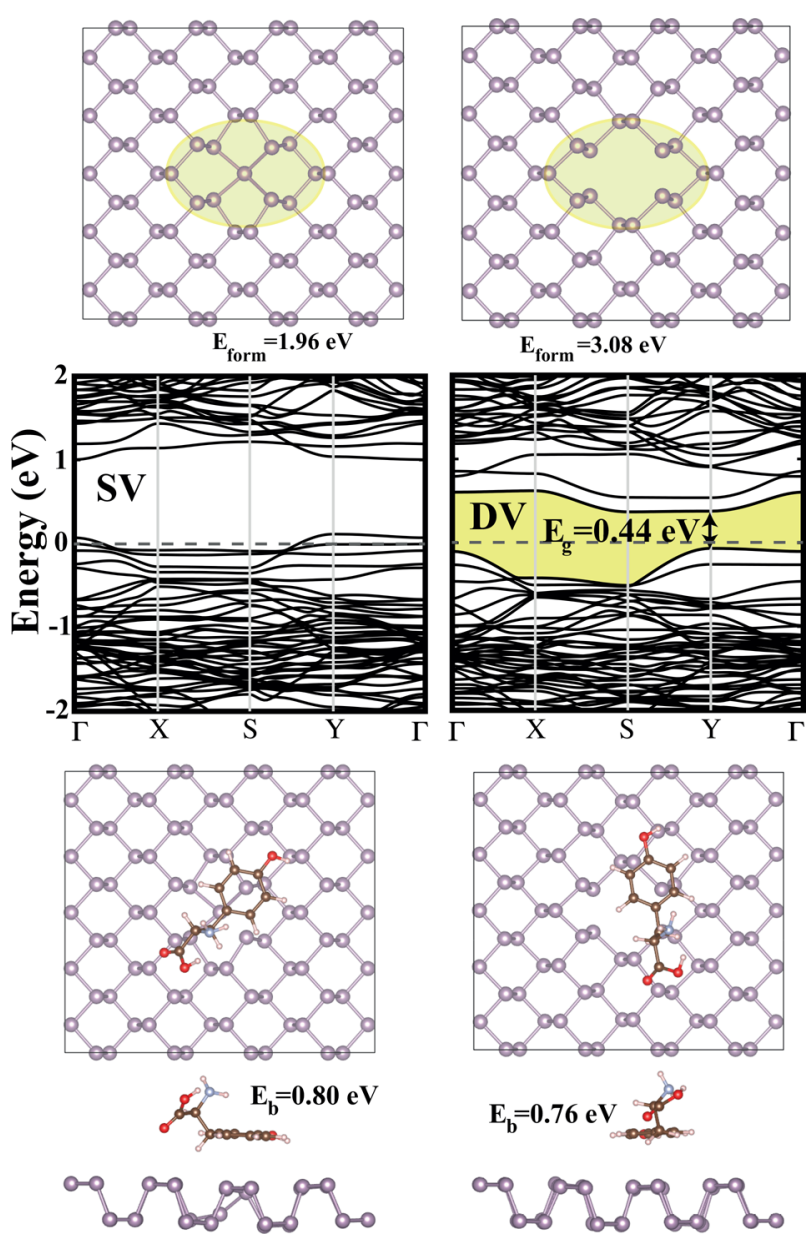

Figure 9. (left) Top view of the atomic structure of single vacancy $(\mathrm{SV})$ created in a $(4 \times 5)$ supercell, energy band structure of the $(4 \times$ 5 ) single vacancy patterned $\alpha$-P monolayer showing a metallic state, and top and side views after the adsorption of tyrosine. (right) Top view of the optimized atomic structure of a divacancy (DV) created in a $(4 \times 5)$ supercell, the energy band structure of divacancy patterned $\alpha-\mathrm{P}$ monolayer showing a semiconducting state with a band gap narrower than that of bare $\alpha$-P-monolayer and top and side views after the physisorption of tyrosine.

\section{ABSORBANCE OF AMINO ACID MOLECULE AND NUCLEOBASES ADSORBED TO $\alpha$-P MONOLAYER}

Recent studies have shown that the optical properties of $2 \mathrm{D}$ ultrathin layers can be modified through their coverage by specific adsorbates. Recently, it has been predicted that graphene undergoes a metal-insulator transition and concomitantly attains three times higher solar absorbance, when coated with glycine. ${ }^{44,74}$ A bare $\alpha$-P monolayer displays electronic properties that are rather different from those of graphene. Additionally, it can provide diverse functionalizations via the adsorption of organic molecules. Our objective is to reveal how the absorbance of the bare as well as $\mathrm{Au}$ atom patterned $\alpha$-P monolayers can be modified with the adsorption of amino acids or nucleobases for possible photovoltaic applications. To this end, we examined the interaction of amino acid or nucleobase covered $\alpha$-P with electromagnetic radiation in terms of the imaginary part of the dielectric function $\left(\epsilon_{2}\right)^{75}$ and absorbance $(A)^{76}$ as a function of photon energy $(\hbar \omega)$. Since the wavelength of the light is always much longer than the interatomic distances, the interaction of light and matter is averaged over many unit cells. ${ }^{77}$ Here we consider one amino acid and two nucleobase molecules, namely, tyrosine, cytosine, and guanine, and their equilibrium $(4 \times 5)$ patterned coverage to the bare $\alpha-\mathrm{P}$, as well as $\mathrm{Au}$ coadsorbed $\alpha-\mathrm{P}$ as described in Figure 6. We calculated the imaginary part of the dielectric constant, $\epsilon_{2}$, and the absorbance, $A$, as a function of the photon energy for the inplane (namely, along the armchair, $\mathbf{E} \| x$, and along the zigzag, $\mathbf{E} \| y$, directions), as well as perpendicular (E $\| z$ direction), electric field polarizations. For the sake of comparison, $\epsilon_{2}$ and $A$ curves of the bare $\alpha$-P monolayer calculated as a function of photon energy are also given. Our results are shown in Figure 10.

The calculated $\epsilon_{2}$ curve of bare phosphorene agrees with that calculated earlier. ${ }^{78}$ Because of high computational cost, excitonic effects are not included in the present study. However, it can be seen that excitonic effects do not highly alter the trend of $\epsilon_{2}$ in the bare phosphorene system when comparing our results with those previously reported. ${ }^{79,80}$ The calculated variation of $\epsilon_{2}$ with photon energy, $\hbar \omega$, presented in Figure 10, reveals that the imaginary part of the dielectric constant of the bare $\alpha$-P is not affected by the $(4 \times 5)$ patterned coverage of one of the three molecules cytosine, guanine, or tyrosine. However, $\epsilon_{2}$ is modified in the energy range, $0<\hbar \omega<\sim 1 \mathrm{eV}$, when one of these three molecules is adsorbed to a $\mathrm{Au}$ atom covering the $\alpha$-P according to the $(4 \times$ 5) pattern.

Lateral (in-plane) and vertical anisotropy of the bare $\alpha$-P monolayer crystal is reflected in the corresponding $A$ curves. The onset of absorption is in compliance with the electronic structure of the $\alpha$-P monolayer and starts at about $0.8 \mathrm{eV}$ for the armchair direction and shifts to 1 and $2 \mathrm{eV}$ for zigzag and perpendicular directions, respectively. The spectrum of the $\mathrm{AA} / \mathrm{NB}+\alpha$-P systems shows similar behaviors to that of the bare $\alpha$-P monolayer for $x$ and $y$ polarizations for photon energies $\hbar \omega<3.0 \mathrm{eV}$. However, for photon energies $3<\hbar \omega<$ $5.0 \mathrm{eV}$, the absorbance is enhanced by $100 \%$ upon the adsorption of amino acids. For vertical polarization in the energy range of $4<\hbar \omega<8 \mathrm{eV}$, the spectra of absorbance, $A$, deviate from those of the bare $\alpha$-P, as well as from in-plane polarization of $\alpha-\mathrm{P}+\mathrm{AA} / \mathrm{NB}$ or $\alpha-\mathrm{P}+\mathrm{Au}_{1}+\mathrm{AA} / \mathrm{NB}$, where the HOMO-LUMO gap region of the AA or NB molecule becomes pronounced due to significant thickness of the adsorbates. In this energy range, the absorbance acquires its maximum value. As compared to $\alpha-\mathrm{P}+$ cytosine and $\alpha-\mathrm{P}+$ tyrosine, the enhancement of absorbance of $\alpha-\mathrm{P}+$ guanine is relatively low. The absorbance is further enhanced when guanine is adsorbed to a Au atom, which in turn, is coadsorbed to the bare $\alpha$-P according to the $(4 \times 5)$ pattern. The effect of coadsorbed $\mathrm{Au}$ atom on the absorbance is, however, reversed in $\alpha-\mathrm{P}+\mathrm{Au}_{1}+$ tyrosine. On the other hand, there is only minute differences between the absorbance spectra of $\alpha-\mathrm{P}+$ cytosine and $\alpha-\mathrm{P}+\mathrm{Au}_{1}+$ cytosine.

\section{DISCUSSIONS AND CONCLUSIONS}

We showed that the amino acid and nucleobase molecules, glutamine, histidine, tyrosine, adenine, guanine, cytosine, and thymine, have significant cohesive energy and wide HOMOLUMO gap ranging between 3 and $5 \mathrm{eV}$. The interaction of these molecules with the bare $\alpha$-P monolayer is weak, and their binding energies range between 0.65 and $0.84 \mathrm{eV}$, which consist of mainly weak van der Waals attraction and weak chemical interaction involving minute exchange of electronic charge. All of these molecules, except cytosine, prefer atomic 

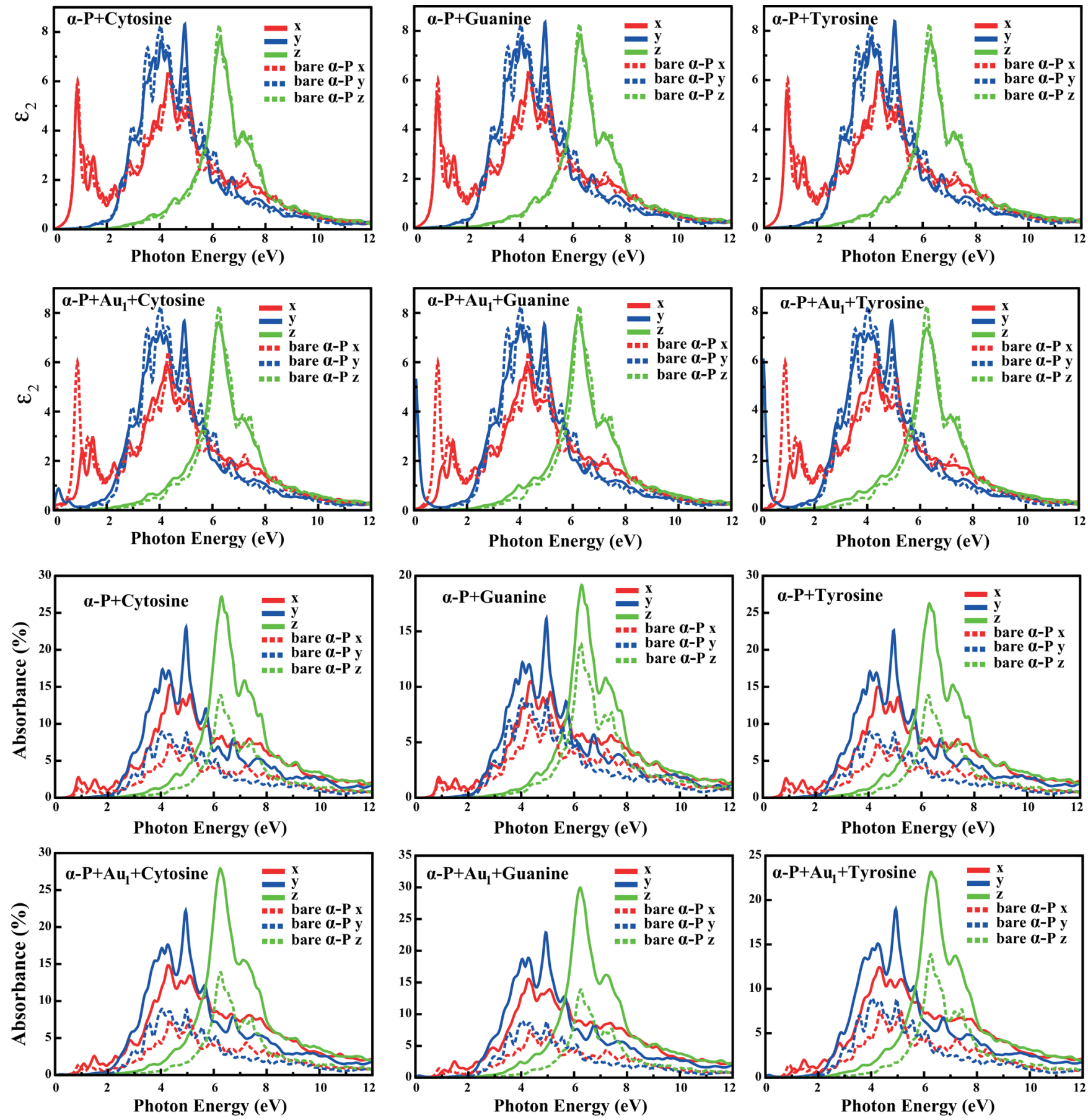

Figure 10. (left, panels 1 and 3) Imaginary part of the dielectric constant $\left(\epsilon_{2}\right)$ and absorbance $(A)$ versus the photon energy, $\hbar \omega$, of cytosine adsorbed to the bare $\alpha$-P according to the $(4 \times 5)$ pattern (as described in Figure 3, named as $\alpha$-P + cytosine. (left, panels 2 and 4$) \epsilon_{2}$ and $A$ versus $\hbar \omega$ of cytosine adsorbed to Au atom, which, in turn, is adsorbed to the bare $\alpha$-P according to the $(4 \times 5)$ pattern (as described in Figure 4 and 6$)$ named as $\alpha-\mathrm{P}+\mathrm{Au}_{1}+$ cytosine. (middle) Same as left panels for adsorbed guanine. (right) Same as left panels for adsorbed tyrosine. $A$ versus photon energy curves of the bare $\alpha$-P monolayer are shown by dashed lines for comparison. $x, y$, and $z$ indicate the polarization directions of the electric field $\mathbf{E}$ as shown by insets.

configurations that are parallel to the $\alpha$-P monolayer. Because of weak binding, their minimum distance from the $\alpha$-P monolayer is $\sim 3 \AA$. Accordingly, the electronic structures of both adsorbed amino acid and nucleobase molecules, and the $\alpha$-P substrate are not affected in any significant manner. The molecules adsorbed within the $(4 \times 5)$ supercell geometry prefer well-defined equilibrium configuration despite very weak coupling with nearest neighbors. This situation indicates that adsorbed molecules can display self-assembly properties for patterned coverage with relatively smaller distance between nearest neighbors.

To mediate strong bonding of these molecules to $\alpha$-P monolayer, we considered adsorbates like gold clusters or $\mathrm{Ti}$ atom on the bare $\alpha$-P monolayer, which may act as interfaces by forming strong bonds both with the bare $\alpha$-P monolayer and the amino acid or nucleobase molecules. In fact, gold 
clusters are bound to $\alpha$-P monolayer with strong bonds and give rise to important modifications in the electronic structure; depending on the size of the cluster and coverage the $\alpha$-P monolayer can attain metallicity and even permanent magnetic moments. When adsorbed to gold clusters on the $\alpha$-P monolayer, the amino acid and nucleobase molecules acquire enhanced binding and modify the electronic structure. Coadsorbed gold clusters, with their size and coating pattern on the monolayer, provide diverse alternatives for fixing individual or self-assembled amino acid or nucleobase molecules and for tuning the electronic structure of combined system.

Like gold clusters, the Ti adatom forms a strong bond with the bare $\alpha$-P monolayer; the spins are polarized to attain permanent magnetic moment, and the width of the band gap can be reduced and undergoes direct-indirect transition depending on the coverage. Hence, the Ti coverage in different patterns on the bare $\alpha$-P monolayer offers an efficient mechanism to attribute magnetic properties to the $\alpha$-P monolayer. In view of its importance, we considered only the interaction between tyrosine and $\mathrm{Ti}$ atom. When adsorbed to the coadsorbed $\mathrm{Ti}$ adatom, the tyrosine molecule attains a strong bond and modifies the electronic properties of the system.

In contrast to earlier predictions that glycine establishes strong bonding with graphene at its defect sites, ${ }^{44}$ the binding of tyrosine to the single vacancy and divacancy sites of the $\alpha$-P monolayer remains weak due to its binding configuration parallel to the monolayer. Nonetheless, cytosine, which prefers perpendicular configurations on the $\alpha$-P monolayer is expected to make relatively stronger bonds with the unsaturated dangling bonds of $\mathrm{P}$ atoms surrounding the vacancy.

The bare $\alpha$-P monolayer by itself is shown to display rather anisotropic optical properties due to its anisotropic geometric structure. When coated by amino acid or nucleobase molecules, the bare and metal adsorbate patterned $\alpha$-P monolayers attain thickness and enhanced absorbance. Hence, not only electronic properties but also optical properties become affected with the adsorption of specific amino acid or nucleobase molecules for specific polarizations of the electric field. We showed that the absorbance of the $\alpha$-P monolayer is enhanced $\sim 100 \%$ when coated with one of three amino acid or nucleobase molecules, namely, cytosine, guanine, or tyrosine. However, the effect of coadsorbed adatoms on the absorbance of amino acid or nucleobase coated $\alpha$-P monolayer is different for different molecules.

In summary, when adsorbed, the amino acids and nucleobases considered in this study can cause diverse modifications of the electronic and optical properties of the bare $\alpha$-P monolayer. Their effects on the physical properties and binding energy can be even more enhanced if they become bound to patterned gold clusters and Ti adatoms on the $\alpha$-P monolayer.

\section{ASSOCIATED CONTENT}

\section{S Supporting Information}

The Supporting Information is available free of charge on the ACS Publications website at DOI: 10.1021/acs.jpcc.9b04713.

Band structures of molecule adsorbed to $\alpha$-P monolayer, $\mathrm{Au}_{x}$ adsorbed to $\alpha$-P monolayer, and defect-containing systems and DOS graphs (PDF)

\section{AUTHOR INFORMATION}

\section{Corresponding Authors}

*E-mail: ethem.akturk@adu.edu.tr. Phone: +9025621308351894. Fax: +902562135379.

*E-mail: ciraci@fen.bilkent.edu.tr. Phone: +903122901216. Fax: +903122664579.

ORCID $\odot$

Ethem Aktürk: 0000-0002-1615-7841

Notes

The authors declare no competing financial interest.

\section{ACKNOWLEDGMENTS}

The computational resources are provided by TUBITAK ULAKBIM, High Performance and Grid Computing Center (TR-Grid e-Infrastructure). S.C. acknowledges financial support from the Academy of Sciences of Turkey (TÜBA).

\section{REFERENCES}

(1) Ren, H.; Kulkarni, D. D.; Kodiyath, R.; Xu, W.; Choi, I.; Tsukruk, V. V. Competitive Adsorption of Dopamine and Rhodamine $6 \mathrm{G}$ on the Surface of Graphene Oxide. ACS Appl. Mater. Interfaces 2014, 6, 2459.

(2) Chen, D.; Li, L.; Guo, L. An environment-friendly preparation of reduced graphene oxide nanosheets via amino acid. Nanotechnology 2011, 22, 325601.

(3) Rubio-Pereda, P.; H. Cocoletzi, G. Density functional theory calculations of biomolecules adsorption on phosphorene for biomedical applications. Appl. Surf. Sci. 2018, 427, 1227.

(4) Cortés-Arriagada, D. Phosphorene as a Template Material for Physisorption of DNA/RNA Nucleobases and Resembling of Base Pairs: A Cluster DFT Study and Comparisons with Graphene. J. Phys. Chem. C 2018, 122, 4870.

(5) Zhang, H. P.; Lin, X. Y.; Lu, X.; Wang, Z.; Fang, L.; Tang, Y. Understanding the interfacial interactions between dopamine and different graphenes for biomedical materials. Materials Chemistry Frontiers 2017, 1, 1156.

(6) Kalantar-zadeh, K.; Ou, J. Z. Biosensors Based on TwoDimensional MoS2. ACS Sens 2016, 1, 5.

(7) Huang, K.; Li, Z.; Lin, J.; Han, G.; Huang, P. Two-dimensional transition metal carbides and nitrides (MXenes) for biomedical applications. Chem. Soc. Rev. 2018, 47, 5109.

(8) Zhao, H.; Ding, R.; Zhao, X.; Li, Y.; Qu, L.; Pei, H.; Yildirimer, L.; Wu, Z.; Zhang, W. Graphene-based nanomaterials for drug and/or gene delivery, bioimaging, and tissue engineering. Drug Discovery Today 2017, 22, 1302.

(9) Suvarnaphaet, P.; Pechprasarn, S. Graphene-Based Materials for Biosensors: A Review. Sensors 2017, 17, 2161.

(10) Shipway, A. N.; katz, E.; Willner, I. Nanoparticle Arrays on Surfaces for Electronic, Optical, and Sensor Applications. ChemPhysChem 2000, 1, 18.

(11) Lee, W. H.; Park, J.; Sim, S. H.; Lim, S.; Kim, K. S.; Hong, B. H.; Cho, K. Surface-Directed Molecular Assembly of Pentacene on Monolayer Graphene for High-Performance Organic Transistors. J. Am. Chem. Soc. 2011, 133, 4447.

(12) MacLeod, J. M.; Rosei, F. Molecular Self-Assembly on raphene. Small 2014, 10, 1038.

(13) Katoch, J.; Kim, S. N.; Kuang, Z.; Farmer, B. L.; Naik, R. R.; Tatulian, S. A.; Ishigami, M. Structure of a Peptide Adsorbed on Graphene and Graphite. Nano Lett. 2012, 12, 2342.

(14) Rahsepar, F. R.; Leung, K. T. Biohybridization of Supported Gold Nanoassemblies on Silicon. J. Phys. Chem. C 2018, 122, 16113.

(15) Ourdjini, Q.; Pawlak, R.; Abel, M.; Clair, S.; Chen, L.; Bergeon, N.; Sassi, M.; Oison, V.; et al. Substrate-mediated ordering and defect analysis of a surface covalent organic framework. Phys. Rev. B: Condens. Matter Mater. Phys. 2011, 84, 125421. 
(16) Radoicic, M. B.; Jankovic, I. A.; Despotovic, V. N.; Sojic, D. V.; Savic, T. D.; Saponjic, Z. V.; Abramovic, B. F.; Comor, M. I. The role of surface defect sites of titania nanoparticles in the photocatalysis: Aging and modification. Appl. Catal., B 2013, 138, 122.

(17) Berney, H.; West, J.; Haefele, E.; Alderman, J.; Lane, W.; Collins, J. A DNA diagnostic biosensor: development, characterisation and performance. Sens. Actuators, B 2000, 68, 100.

(18) Sanvicens, N.; Pastells, C.; Pascual, N.; Marco, M.-P. Nanoparticle-based biosensors for detection of pathogenic bacteria. TrAC, Trends Anal. Chem. 2009, 28, 1243.

(19) Farimani, A. B.; Min, K.; Aluru, N. R. DNA Base Detection Using a Single-Layer $\mathrm{MoS}_{2}$. ACS Nano 2014, 8, 7914.

(20) Xiao, L.; Gao, C.; Zhang, Y.; Yao, Q.; Zhang, G.-J.; Xu, L. A $\mathrm{MoS}_{2}$ Nanosheet-Based Fluorescence Biosensor for Simple and Quantitative Analysis of DNA Methylation. Sensors 2016, 16, 1561.

(21) Feng, S.; et al. Tunable excitonic emission of monolayer $W S_{2}$ for the optical detection of DNA nucleobases. Nano Res. 2018, 11, 1744.

(22) Lee, J.-H.; Choi, Y.-K.; Kim, H.-J.; Scheicher, R. H.; Cho, J.-H. Physisorption of DNA Nucleobases on hâǍKBN and Graphene: vdWCorrected DFT Calculations. J. Phys. Chem. C 2013, 117, 13435.

(23) Janani, K.; Thiruvadigal, D. J. Adsorption of Essential Minerals on L-Glutamine Functionalized Zigzag Graphene Nanoribbon-A First Principles DFT Study. Appl. Surf. Sci. 2018, 449, 829.

(24) Li, Z.; Chen, Y.; Li, X.; Kamins, T. I.; Nauka, K.; Williams, R. S. Sequence-Specific Label-Free DNA Sensors Based on Silicon Nanowires. Nano Lett. 2004, 4, 245.

(25) Dragneva, N.; Rubel, O.; Floriano, W. B. Molecular Dynamics of Fibrinogen Adsorption onto Graphene, but Not onto Poly(ethylene glycol) Surface, Increases Exposure of Recognition Sites That Trigger Immune Response. J. Chem. Inf. Model. 2016, 56, 706.

(26) Dong, S.; Zeng, M.; Wang, D.; Liu, Z.; Zhao, Y.; Yang, H. Antioxidant and biochemical properties of protein hydrolysates prepared from Silver carp (Hypophthalmichthys molitrix). Food Chem. 2008, 107, 1485.

(27) Liang, G.; Yang, Z.; Zhang, R.; Li, L.; Fan, Y.; Kuang, Y.; Gao, Y.; Wang, T.; Lu, W. W.; Xu, B. Supramolecular Hydrogel of a dAmino Acid Dipeptide for Controlled Drug Release in Vivo. Langmuir 2009, 25, 8419.

(28) Wang, E. A.; Rosen, V.; D’Alessandro, J. S.; Bauduy, M.; Cordes, P.; Harada, T.; Israel, D. I.; Hewick, R. M.; Kerns, K. M.; LaPan, P. Recombinant human bone morphogenetic protein induces bone formation. Proc. Natl. Acad. Sci. U. S. A. 1990, 87, 2220.

(29) Johnston, G. A. R.; Davies, L. P. Postnatal changes in the high affinity uptake of glycine and GABA in the rat cental nervous system. J. Neurochem. 1974, 22, 101.

(30) Ersan, F.; Üzengi Aktürk, O.; Aktürk, E.; Ciraci, S. MetalInsulator Transition and Heterostructure Formation by Glycines SelfAssembled on Defect-Patterned Graphene. J. Phys. Chem. C 2018, 122, 14598.

(31) Ersan, F.; Aktürk, E.; Ciraci, S. Glycine self-assembled on graphene enhances the solar absorbance performance. Carbon 2019, 143, 329.

(32) Ullrich, A.; Schlessinger, J. Signal Transduction by Receptors with Tyrosine Kinase Activity. Cell 1990, 61, 203.

(33) Pazdrak, K.; Schreiber, D.; Forsythe, P.; Justement, L.; Alam, R. The intracellular signal transduction mechanism of interleukin 5 in eosinophils: the involvement of lyn tyrosine kinase and the Ras-Raf-1MEK-microtubule-associated protein kinase pathway. J. Exp. Med. 1995, 181, 1827.

(34) Weiss, A. T. Cell Antigen Receptor Signal Transduction: A Tale of Tails and Cytoplasmic Protein-Tyrosine Kinases. Cell 1993, 73, 209.

(35) Nie, R.; Bo, X.; Wang, H.; Zeng, L.; Guo, L. Chiral electrochemical sensing for tyrosine enantiomers on glassy carbon electrode modified with cysteic acid. Electrochem. Commun. 2013, 27, 112.

(36) Liang, H.-J.; Ling, T.-R.; Rick, J. F.; Chou, T.-C. Molecularly imprinted electrochemical sensor able to enantroselectivly recognize D and L-tyrosine. Anal. Chim. Acta 2005, 542, 83.
(37) Biswas, A.; Banerjee, S.; Gart, E. V.; Nagaraja, A. T.; McShane, M. J. Gold Nanocluster Containing Polymeric Microcapsules for Intracellular Ratiometric Fluorescence Biosensing. ACS Omega 2017, 2, 2499-2506.

(38) Wang, G.; Huang, T.; Murray, R. W.; Menard, L.; Nuzzo, R. G. Near-IR Luminescence of Monolayer-Protected Metal Clusters. J. Am. Chem. Soc. 2005, 127, 812-813.

(39) Wu, Z.; Jin, R. On the Ligand's Role in the Fluorescence of Gold Nanoclusters. Nano Lett. 2010, 10, 2568-2573.

(40) Carnerero, J. M.; Sanchez-Coronilla, A.; Martin, E. I.; JimenezRuiz, A.; Prado-Gotor, R. Quantification of nucleobases/gold nanoparticles interactions: energetics of the interactions through apparent binding constants determination. Phys. Chem. Chem. Phys. 2017, 19, 22121.

(41) Elghanian, R.; Storhoff, J. J.; Mucic, R. C.; Mirkin, C. A.; Letsinger, R. L. Selective Colorimetric Detection of Polynucleotides Based on the Distance-Dependent Optical Properties of Gold Nanoparticles. Science 1997, 277, 1078.

(42) Nowicka, A. M.; Fau, M.; Rapecki, T.; Donten, M. Polypyrrole$\mathrm{Au}$ Nanoparticles Composite as Suitable Platform for DNA Biosensor with Electrochemical Impedance Spectroscopy Detection. Electrochim. Acta 2014, 140, 65.

(43) Li, Y.; Schluesener, H. J.; Xu, S. Gold nanoparticle-based biosensors. Gold Bulletin 2010, 43, 29.

(44) Ersan, F.; Üzengi Aktürk, O.; Aktürk, E.; Ciraci, S. MetalInsulator Transition and Heterostructure Formation by Glycines SelfAssembled on Defect-Patterned Graphene. J. Phys. Chem. C 2018, 122, 14598.

(45) Kresse, G.; Furthmüller, J. Efficient iterative schemes for ab initio total-energy calculations using a plane-wave basis set. Phys. Rev. B: Condens. Matter Mater. Phys. 1996, 54, 11169.

(46) Kresse, G.; Furthmüller, J. Efficiency of ab-initio total energy calculations for metals and semiconductors using a plane-wave basis set. Comput. Mater. Sci. 1996, 6, 15.

(47) Perdew, J. P.; Burke, K.; Ernzerhof, M. Generalized gradient approximation made simple. Phys. Rev. Lett. 1996, 77, 3865.

(48) Blöchl, P. E. Projector augmented-wave method. Phys. Rev. B: Condens. Matter Mater. Phys. 1994, 50, 17953.

(49) Kresse, G.; Joubert, D. From ultrasoft pseudopotentials to the projector augmented-wave method. Phys. Rev. B: Condens. Matter Mater. Phys. 1999, 59, 1758.

(50) Grimme, S. Semiempirical GGA-type density functional constructed with a long-range dispersion correction. J. Comput. Chem. 2006, 27, 1787-1799.

(51) Monkhorst, H. J.; Pack, J. D. Special points for Brillouin-zone integrations. Phys. Rev. B 1976, 13, 5188.

(52) Paier, J.; Marsman, M.; Hummer, K.; Kresse, G.; Gerber, I.; Angyan, J. Screened hybrid density functionals applied to solids. J. Chem. Phys. 2006, 124, 154709.

(53) Heyd, J.; Scuseria, G. E. Efficient hybrid density functional calculations in solids: Assessment of the Heyd-Scuseria-Ernzerhof screened Coulomb hybrid functional. J. Chem. Phys. 2004, 121, 1187.

(54) Henkelman, G.; Arnaldsson, A.; Jónsson, H. A fast and robust algorithm for Bader decomposition of charge density. Comput. Mater. Sci. 2006, 36, 354-360.

(55) Wise, D. R.; Thompson, C. B. Glutamine addiction: a new therapeutic target in cancer. Trends Biochem. Sci. 2010, 35, 427.

(56) Gomes, L. C.; Carvalho, A. Phosphorene analogues: Isoelectronic two-dimensional group-IV monochalcogenides with orthorhombic structure. Phys. Rev. B: Condens. Matter Mater. Phys. 2015, 92, 085406.

(57) Cakir, D.; Sahin, H.; Peeters, F. M. Tuning of the electronic and optical properties of single-layer black phosphorus by strain. Phys. Rev. B: Condens. Matter Mater. Phys. 2014, 90, 205421.

(58) Li, L.; Yu, Y.; Ye, G. J.; Ge, Q.; Ou, X.; Wu, H.; Feng, D.; Chen, X. H.; Zhang, Y. Black phosphorus field-effect transistors. Nat. Nanotechnol. 2014, 9, 372. 
(59) Liu, H.; Neal, A. T.; Zhu, Z.; Luo, Z.; Xu, X.; Tománek, D.; Ye, P. D. Phosphorene: An Unexplored 2D Semiconductor with a High Hole Mobility. ACS Nano 2014, 8, 4033.

(60) Xia, F.; Wang, H.; Jia, Y. Rediscovering black phosphorus as an anisotropic layered material for optoelectronics and electronics. Nat. Commun. 2014, 5, 4458.

(61) He, J.; He, D.; Wang, Y.; Cui, Q.; Bellus, M. Z.; Chiu, H.-Y.; Zhao, H. Exceptional and Anisotropic Transport Properties of Photocarriers in Black Phosphorus. ACS Nano 2015, 9, 6436.

(62) Ong, Z. Y.; Cai, Y.; Zhang, Y.-W.; Zhang, G. Strong Thermal Transport Anisotropy and Strain Modulation in Single-Layer Phosphorene. J. Phys. Chem. C 2014, 118, 25272.

(63) Luo, Z.; Maassen, J.; Du, Y.; Garrelts, R. P.; Lundstrom, M. S.; Ye, P. D.; Xu, X.; Deng, Y. Anisotropic in-plane thermal conductivity observed in few-layer black phosphorus. Nat. Commun. 2015, 6, 8572. (64) Cortés-Arriagada, D.; Ortega, D. E. Effects on the aromatic character of DNA/RNA nucleobases due to its adsorption onto graphene. Int. J. Quantum Chem. 2018, 118, No. e25699.

(65) Wang, W.; Bao, T.; Zeng, X.; Xiong, H.; Wen, W.; Zhang, X.; Wang, S. Ultrasensitive electrochemical DNA biosensor based on functionalized gold clusters/graphene nanohybrids coupling with exonuclease III-aided cascade target recycling. Biosens. Bioelectron. 2017, 91, 183.

(66) Chen, M.; Hou, C.; Huo, D.; Bao, J.; Fa, H.; Shen, C. An electrochemical DNA biosensor based on nitrogen-doped graphene/ $\mathrm{Au}$ nanoparticles for human multidrug resistance gene detection. Biosens. Bioelectron. 2016, 85, 684.

(67) Huang, H.; Bai, W.; Dong, C.; Guo, R.; Liu, Z. An ultrasensitive electrochemical DNA biosensor based on graphene/Au nanorod/ polythionine for human papillomavirus DNA detection. Biosens. Bioelectron. 2015, 68, 442.

(68) Deka, A.; Deka, R. C. Structural and electronic properties of stable $\mathrm{Au}_{n}(\mathrm{n}=2-13)$ clusters: A density functional study. J. Mol. Struct.: THEOCHEM 2008, 870, 83.

(69) Häkkinen, H.; Landman, U. Gold clusters $A_{u N} N_{2} \mathrm{~N}_{10}$ and their anions. Phys. Rev. B: Condens. Matter Mater. Phys. 2000, 62, 2287.

(70) Ersan, F.; Aktürk, E.; Ciraci, S. Interaction of Adatoms and Molecules with Single-Layer Arsenene Phases. J. Phys. Chem. C 2016, 120, 14345-14355.

(71) Üzengi Aktürk, O.; Aktürk, E.; Ciraci, S. Effects of adatoms and physisorbed molecules on the physical properties of antimonene. Phys. Rev. B: Condens. Matter Mater. Phys. 2016, 93, 035450.

(72) Pan, Y.; Wang, Y.; Ye, M.; Quhe, R.; Zhong, H.; Song, Z.; Peng, X.; Yu, D.; Yang, J.; Shi, J.; Lu, J. Monolayer Phosphorene-Metal Contacts. Chem. Mater. 2016, 28, 2100.

(73) Liu, J.; Qiao, M.; Zhu, X.; Jing, Y.; Li, Y. Ti2PTe2 monolayer: a promising two-dimensional anode material for sodium-ion batteries. RSC Adv. 2019, 9, 15536.

(74) Ersan, F.; Aktürk, E.; Ciraci, S. Glycine self-assembled on graphene enhances the solar absorbance performance. Carbon 2019, 143, 329.

(75) Gajdos, M.; hummer, K.; Kresse, G.; Furthmüller, J.; Bechstedt, F. Linear optical properties in the projector-augmented wave methodology. Phys. Rev. B: Condens. Matter Mater. Phys. 2006, 73, 045112 .

(76) Tan, T. L.; Ng, M.-F.; Eda, G. Stable Monolayer Transition Metal Dichalcogenide Ordered Alloys with Tunable Electronic Properties. J. Phys. Chem. C 2016, 120, 2501.

(77) Suresh, S. Investigation of the Optical and Dielectric Properties of the Urea L-malic Acid NLO Single Crystal. Am. Chem. Sci. J. 2013, 3,325 .

(78) Mahabal, M. S.; Deshpande, M. D.; Hussain, T.; Ahuja, R. Sensing Characteristics of Phosphorene Monolayers toward $\mathrm{PH} 3$ and AsH3 Gases upon the Introduction of Vacancy Defects. J. Phys. Chem. C 2016, 120, 20428.

(79) Ferreira, F.; Ribeiro, R. M. Improvements in the GW and Bethe-Salpeter-equation calculations on phosphorene. Phys. Rev. B: Condens. Matter Mater. Phys. 2017, 96, 115431.
(80) Nguyen-Truong, H. T. Energy-loss function for monolayer phosphorene. J. Mater. Sci. 2018, 53, 15541. 